\title{
Future constraints on dynamical dark-energy using gravitational-wave standard sirens
}

\author{
Minghui Du, ${ }^{1, *}$ Weiqiang Yang, ${ }^{2, \dagger}$ Lixin Xu, ${ }^{1, \pitchfork}$ Supriya Pan, ${ }^{3, \S}$ and David F. Mota ${ }^{4, \|}$ \\ ${ }^{1}$ Institute of Theoretical Physics, School of Physics, Dalian University of Technology, \\ Dalian, 116024, People's Republic of China \\ ${ }^{2}$ Department of Physics, Liaoning Normal University, Dalian, 116029, People's Republic of China \\ ${ }^{3}$ Department of Mathematics, Presidency University, 86/1 College Street, Kolkata 700073, India \\ ${ }^{4}$ Institute of Theoretical Astrophysics, University of Oslo, 0315 Oslo, Norway
}

(Received 24 December 2018; published 23 August 2019)

\begin{abstract}
The detection of gravitational waves (GW) by the LIGO and Virgo collaborations offers a whole new range of possible tests and opens up a new window that may shed light on the nature of dark energy and dark matter. In the present work we investigate how future gravitational-wave data could help to constrain different dynamical dark energy models. In particular, we perform cosmological forecastings of a class of well-known and most used dynamical dark energy models using the third-generation gravitational wave detector, the Einstein Telescope. We have considered 1000 simulated GW events in order to constrain the parameter space of the dynamical dark energy models. Our analyses show that the inclusion of the GW data from the Einstein Telescope significantly improves the parameter space of the dynamical dark energy models compared to their constraints extracted from the standard cosmological probes, namely, the cosmic microwave observations, baryon acoustic oscillations distance measurements, supernove type Ia, and the Hubble parameter measurements.
\end{abstract}

DOI: 10.1103/PhysRevD.100.043535

\section{INTRODUCTION}

According to the latest claims by LIGO and Virgo collaborations, the gravitational waves $(\mathrm{GW})$ from a pair of two very massive black holes around 36 and 29 solar masses have been detected, known as the GW150914 event [1]. Subsequently, the investigations in a series of further works [2-6] also claimed similar detection. Just after the detection of GW from binary black holes, again GW from a binary neutron star merger (known as the GW170817 event [7]) together with an electromagnetic counterpart known as GRB 170817A event [8] was also detected. Without any doubt, the detection of GW, if we avoid its counterattacks, is an appreciable event for modern cosmology that naturally thrilled the scientific community offering some new insights in the physics of dark energy and modified gravity theories at the fundamental level. Following this a lot of investigations have already been performed by many researchers in order to understand how GW could affect the cosmological theories of interest; see for instance [9-29]. One of the most important properties is that GW propagate practically with the light speed, as reported by

\footnotetext{
*angelbeats@mail.dlut.edu.cn

†11102004@163.com

*1xxu@dlut.edu.cn

\$supriya.maths@presiuniv.ac.in

"mota@astro.uio.no
}

the events GW170817 [7] and GRB 170817A [8]. Thus, by using the extracted properties from GW, for instance, its propagation speed, one can impose strong constraints on the cosmological models as well as exclude some cosmological theories. Especially, GW data provide a novel approach of luminosity distance measurements, known as standard sirens.

Motivated by the earlier investigations, in the present work, we focus on the dynamical dark energy cosmologies through their parametrizations with an aim to examine how luminosity distances extracted from future GW data could affect the bounds on the aforementioned dark energy models. The parametrizations of the dark energy sector are a well-motivated area in cosmology where the primary content is the dark energy equation of state defined by $w_{x}=p_{x} / \rho_{x}$, in which $p_{x}, \rho_{x}$ are respectively the pressure and energy density of the dark energy fluid. We note that in the context of modified gravity theories, such parametrizations can be viewed in terms of an effective dark energy equation of state. Alternatively, using a different dark energy equation of state (in the context of Einstein's gravity theory) or effective dark energy equation of state (in the context of modified gravitational theories), one could be able to trace the expansion history of the Universe, and test them using the observational data. In this work we consider that the underlying gravitational theory is described by Einstein's gravity and the large scale structure 
of our Universe is homogeneous and isotropic; and hence, the geometry of the Universe is described by the Friedmann-Lemaître-Robertson-Walker (FLRW) metric. Now, concerning the dynamical dark energy parametrizations, we recall numerous parametrizations that have been investigated widely with the available observational data [30-48]. Some well-known and most used dark energy parametrizations in this series are the Chevallier-PolarskiLinder (CPL) parametrization [30,31], logarithmic parametrization [35], Jassal-Bagla-Padmanabhan (JBP) parametrization [36], and Barboza-Alcaniz (BA) parametrization [39]. Here, considering these four well-known dark energy parametrzations, namely, CPL, logarithmic, JBP, and BA, we perform a robust analysis by constraining their parameter space using the simulated $\mathrm{GW}$ data from the Einstein Telescope along with the standard astronomical probes such as cosmic microwave background (CMB) radiation [49,50], baryon acoustic oscillations (BAO) [5153], supernove type Ia (SNIa) [54], and Hubble parameter measurements from the cosmic chronometers (CC) [55], in order to see how the data from GW improve the parameter space of these known parametrizations compared to their usual cosmological constraints availed from the known cosmological probes, CMB, BAO, SNIa, and CC. We refer to some earlier works on dark energy with similar motivation, meaning where the simulated GW data from the Einstein Telescope were taken into account [56-61]. We mention that it will also be interesting to use simulated GW data from other observatories like Laser Interferometer Space Antenna [62], Deci-hertz Interferometer Gravitational wave Observatory [63,64], and TianQin [65]. However, in the present work we mainly concentrate on how GW data from one particular source, namely from the Einstein Telescope, could affect a class of well-known and most used dynamical dark energy parametrizations. One can equally apply other GW sources to a specific model in order to compare their constraining power.

However, apart from the GW data, a number of upcoming cosmological surveys, such as, Simons Observatory Collaboration (SOC) [66], Cosmic Microwave Background Stage-4 (CMB-S4) [67], EUCLID Collaboration [68,69], Dark Energy Spectroscopic Instrument (DESI) [70], and Large Synoptic Survey Telescope (LSST) [71-73], are all dedicated to explore more about the nature of the dark sector of our Universe and to provide more precise constraints on the dark energy equation of state. So, it is expected that the upcoming cosmological surveys mentioned above will play a crucial role to understand the physics of the dark universe. Along similar lines, it is also important to understand the constraining power of different surveys by investigating the improvements of the cosmological parameters. This will enable us to understand how the Einstein Telescope and other GW observatories perform with respect to other cosmological surveys, such as SOC, CMB-S4, EUCLID, etc. Thus, for a better conclusion about the constraining power between the cosmological surveys, it is important to apply all of them on a specific cosmological model. Such an investigation is truly important in the context of cosmological physics. A systematic and dedicated analysis of the dynamical dark energy models taking all the future cosmological surveys mentioned above is the subject of a forthcoming work.

The work has been structured in the following way. In Sec. II we briefly introduce the background and perturbative evolutions for any dark energy parametrization as well as introducing the parametrizations of our interest. After that in Sec. III we describe the method to simulate the GW data from the Einstein Telescope and show how to use the simulated GW data in order to constrain an underlying theory. In Sec. IV we introduce the standard astronomical probes as well as the methodology for constraining the model parameters. Then in Sec. V we discuss the results of our analyses. Finally, we close the work in Sec. VI with a brief summary of all the results obtained.

\section{DYNAMICAL DARK ENERGY}

In this section we describe the general evolution laws of a dynamical dark energy component at the level of background and perturbations.

It is well known that at large scale, our Universe is perfectly homogeneous and isotropic. Such geometrical description of our Universe is characterized by the FLRW line element given by

$d \mathrm{~s}^{2}=-d t^{2}+a^{2}(t)\left[\frac{d r^{2}}{1-k r^{2}}+r^{2}\left(d \theta^{2}+\sin ^{2} \theta d \phi^{2}\right)\right]$,

where $a(t)$ (hereafter we denote it simply by $a$ ) is the expansion scale factor of the Universe and $k$ is the curvature scalar. For $k=0,+1,-1$, three different geometries, namely, the spatially flat, closed, and the open Universe are described. Further, we assume that the gravitational sector of the Universe is described by the Einstein's general theory of relativity where the total matter sector of the Universe is minimally coupled to the Einstein gravity. This total matter sector comes from radiation, baryons, pressureless dark matter, and dark energy. Thus, with the above information, one can explicitly write down Einstein's field equations as

$$
\begin{gathered}
H^{2}+\frac{k}{a^{2}}=\frac{8 \pi G}{3} \rho_{\mathrm{tot}}, \\
2 \dot{H}+3 H^{2}+\frac{k}{a^{2}}=-8 \pi G p_{\mathrm{tot}},
\end{gathered}
$$

where $H \equiv \dot{a} / a$ is the Hubble factor of the FLRW Universe; $\rho_{\text {tot }}=\rho_{r}+\rho_{b}+\rho_{c}+\rho_{x}$, is the total energy density of the Universe and $p_{\text {tot }}=p_{r}+p_{b}+p_{c}+p_{x}$ is the total pressure coming from the individual fluid. Let us 
note that here $\rho_{i}(i=r, b, c, x)$ and $p_{i}$ are respectively the energy density and the pressure of the $i$ th component where the subscripts $r, b, c, x$ respectively correspond to radiation, baryons, cold dark matter, and the dark energy sector. Now, using the Bianchi's identity, the conservation law for the total fluid follows,

$$
\dot{\rho}_{\text {tot }}+3 H\left(\rho_{\text {tot }}+p_{\text {tot }}\right)=0 .
$$

One can easily find that the conservation equation (4) can be obtained if we simply use the field equations (2) and (3). Since we do not have any interaction between the fluids, the conservation equation of each fluid follows the evolution

$$
\dot{\rho}_{i}+3 H\left(p_{i}+\rho_{i}\right)=0 \Leftrightarrow \dot{\rho}_{i}+3 H\left(1+w_{i}\right) \rho_{i}=0,
$$

where $w_{i}=p_{i} / \rho_{i}$ is the equation of state of the $i$ th fluid and it takes 1/3, 0, 0 for radiation, baryons, and cold dark matter. The equation of state of the dark energy fluid is unknown and in this work we consider that $w_{x}$ has a dynamical character and henceforth we consider some particular expressions for it. We make a final comment regarding the geometrical shape of the Universe. As from the observational sources, the Universe is almost flat [74], and henceforth, throughout the present work we assume $k=0$ in Einstein's field equations (2) and (3). Now, let us get back to the conservation equation (5), from which one can solve the evolution equations for the governing matter components. In particular, the evolution of the dark energy fluid can be written in terms of its energy density as

$$
\rho_{x}=\rho_{x, 0}\left(\frac{a}{a_{0}}\right)^{-3} \exp \left(-3 \int_{a_{0}}^{a} \frac{w_{x}\left(a^{\prime}\right)}{a^{\prime}} d a^{\prime}\right),
$$

where $\rho_{x, 0}$ is the present value of the dark energy density $\rho_{x}$, and here $a_{0}$ is the present value of the scale factor where $1+z=a_{0} / a$. Without any loss of generality we set the present value of the scale factor to be unity; that means $a_{0}=1$. Thus, with the above set of equations, for any prescribed dark energy equation of state, in principle, it is possible to determine the background evolution of the Universe.

However, at the same time, it is important to understand the behavior of the model at the level of perturbations since that enables us to understand the formation of structure of the Universe.

Thus, in order to investigate the cosmological perturbations, we consider the perturbed FLRW metric that takes the following expression,

$$
d s^{2}=a^{2}(\tau)\left[-d \tau^{2}+\left(\delta_{i j}+h_{i j}\right) d x^{i} d x^{j}\right],
$$

where $\tau$ is the conformal time and the quantities $\delta_{i j}, h_{i j}$ respectively denote the unperturbed and the perturbated metric tensors. Now, for the above perturbed metric (7), one can conveniently write the Einstein's equations either in the conformal Newtonian gauge or in the synchronous gauge in the Fourier space $\kappa$. We choose the synchronous gauge and thus using the energy-momentum balance equation $T_{; \nu}^{\mu \nu}=0$, for the $i$ th fluid the continuity and the Euler equations for a mode can be written as [75-77]

$$
\begin{aligned}
\delta_{i}^{\prime}= & -\left(1+w_{i}\right)\left(\theta_{i}+\frac{h^{\prime}}{2}\right)-3 \mathcal{H}\left(\frac{\delta p_{i}}{\delta \rho_{i}}-w_{i}\right) \delta_{i} \\
& -9 \mathcal{H}^{2}\left(\frac{\delta p_{i}}{\delta \rho_{i}}-c_{a, i}^{2}\right)\left(1+w_{i}\right) \frac{\theta_{i}}{\kappa^{2}}, \\
\theta_{i}^{\prime}= & -\mathcal{H}\left(1-3 \frac{\delta p_{i}}{\delta \rho_{i}}\right) \theta_{i}+\frac{\delta p_{i} / \delta \rho_{i}}{1+w_{i}} \kappa^{2} \delta_{i}-\kappa^{2} \sigma_{i},
\end{aligned}
$$

where any prime associated with each variable denotes the differentiation with respect to the conformal time $\tau$; $\delta_{i}=\delta \rho_{i} / \rho_{i}$ is the density perturbation for the $i$ th fluid; $\mathcal{H}=a^{\prime} / a$, is the conformal Hubble factor; $h=h_{j}^{j}$ is the trace of $h_{i j}$, and $\theta_{i} \equiv i \kappa^{j} v_{j}$ is the divergence of the $i$ th fluid velocity. The quantity $c_{a, i}^{2}=\dot{p}_{i} / \dot{\rho}_{i}$ denotes the adiabatic sound speed of the $i$ th fluid whereas $c_{s}^{2}=\delta p_{i} / \delta \rho_{i}$ is the physical sound speed related with another as $c_{a, i}^{2}=$ $w_{i}-\frac{w_{i}^{\prime}}{3 \mathcal{H}\left(1+w_{i}\right)}$. Finally, we note that $\sigma_{i}$ is the anisotropic stress of the $i$ th fluid; however, we neglect its contribution for its minimal contribution as reported by some recent observational data [78].

Now, we close this section by enlisting the dark energy parametrizations that we wish to study in this work. We consider four well-known dark energy parametrizations as follows. The first one is the Chevallier-Polarski-Linder model $[30,31]$ having the following expression,

$$
w_{x}(z)=w_{0}+w_{a} \frac{z}{1+z},
$$

where $w_{0}$ is the present value of $w_{x}(z)$ and $w_{a}=d w_{x}(z) / d z$ at $z=0$ is another free parameter of this model.

As a second model, we consider the logarithmic parametrization introduced by G. Efstathiou [35],

$$
w_{x}(z)=w_{0}+w_{a} \ln (1+z)
$$

where $w_{0}$ and $w_{a}$ parameters have the same meanings as described for the CPL parametrization.

We then consider another dark energy parametrization widely known as the JBP parametrization [36],

$$
w_{x}(z)=w_{0}+w_{a} \frac{z}{(1+z)^{2}},
$$

and here, too, $w_{0}$ and $w_{a}$ parameters have the same meanings as described for the above two models, namely, CPL and logarithmic. 
Finally, we end up with the Barboza-Alcaniz parametrization [39],

$$
w_{x}(z)=w_{0}+w_{a} \frac{z(1+z)}{1+z^{2}},
$$

where $w_{0}, w_{a}$ have the same meanings as described above for other dark energy parametrizations.

\section{METHOD OF SIMULATING GW DATA AND ITS USE}

In this section we describe the method for simulating the Gravitational Waves Standard Sirens (GWSS) data, each data point of which consists of $\left(z, d_{L}(z), \sigma_{d_{L}}\right)$ of a GW source, where $d_{L}(z)$ is the luminosity distance at the redshift $z$ and $\sigma_{d_{L}}$ is the associated error with $d_{L}(z)$. The constraining ability of this catalogue, together with other astronomical datasets, is further investigated in various cosmological models, for instance, $[56,57,60]$. The simulation of GW data is model dependent; thus one needs to choose the fiducial values of model parameters. In this paper, each set of parameters used in the GW simulation is decided by other observational data under a specific cosmological model, and after that the aforementioned GW data as well as the real data from different observational sources are combined to constrain the same model. This procedure has been followed in Sec. V.

The initial step to generate the GWSS data is performed by simulating the redshift distribution of the sources. In this paper we assume the redshifts of all observed GW sources are available. Practically, this is achieved by employing techniques such as identifying the electromagnetic counterparts. Our interest is focused on GW events originating from two types of binary systems: the binary system of a black hole (BH) and a neutron star (NS) identified as BHNS as well as binary neutron star (BNS).

Following some earlier works in this direction [56,57, 60], the redshift distribution of the observable sources is given by

$$
P(z) \propto \frac{4 \pi d_{C}^{2}(z) R(z)}{H(z)(1+z)},
$$

where $d_{C}(z)$ represents the comoving distance at the redshift $z ; R(z)$ is the merger rate of binary system (BHNS or BNS) with the fitting form $[57,79,80]$

$$
R(z)= \begin{cases}1+2 z, & z \leq 1, \\ \frac{3}{4}(5-z), & 1<z<5, \\ 0, & z \geq 5\end{cases}
$$

Based on the prediction of the Advanced LIGO-Virgo network, the detailed configuration of our simulation is as follows. The ratio between observed BHNS and BNS events is set to be 0.03 , which makes BNS the overwhelming majority of GW sources. By roughly considering the mass distribution of the astrophysical objects NS and $\mathrm{BH}$, we perform random sampling of their masses from uniform distributions $U\left(M_{\odot}, 2 M_{\odot}\right)$ and $U\left(3 M_{\odot}, 10 M_{\odot}\right)$, respectively, with $M_{\odot}$ being one solar mass. For more details, we refer to $[57,60]$.

Thus, according to the redshift and mass distribution described above, the catalogue of the GWSS data can be easily obtained through the introduction of the fiducial model, which could be any well-motivated cosmological model. Now, for the spatially flat Universe, technically, one could find the expression for $H(z)$ for the concerned cosmological model and consequently, the luminosity distance $d_{L}(z)$ of the GW sources can now be calculated through the relation

$$
d_{L}(z)=(1+z) \int_{0}^{z} \frac{d z^{\prime}}{H\left(z^{\prime}\right)} .
$$

Hence, the mean luminosity distances of all the GW sources can be generated using Eq. (16). That means the $d_{L}(z)$ vs $z$ relation can be obtained for every GW event for the concerned cosmological model, which as mentioned could be any well-motivated cosmological model. Although in some earlier works, $\Lambda \mathrm{CDM}$ has been considered the fiducial model, in a similar fashion, instead of the $\Lambda \mathrm{CDM}$ model, one may fix some other dark energy models to generate the simulated GW data, since there is no such strict rule to select the $\Lambda \mathrm{CDM}$ model as the fiducial one. In this work we have not fixed $\Lambda \mathrm{CDM}$ as the fiducial model, which is usually done (for instance, see $[56,57])$; rather we have considered the dynamical dark energy models as the fiducial models. We describe this later in more detail.

Now, while measuring the luminosity distance of the GW source, certainly, one needs to calculate the associated error, which we denote by $\sigma_{d_{L}}$. In order to calculate this error, one needs the expression of the GW signal, i.e., the strain of GW interferometers. Note that, since the GW amplitude relies on $d_{L}(z)$, one can extract the information regarding $d_{L}(z)$ once other parameters (e.g., masses of the binary system etc.) are evaluated from the waveform. This is the reason why the GW events are often referred to as the standard sirens, analogous to the supernovae type Ia standard candles. As a consequence, the error of $\mathrm{GW}$ detection (given in terms of GW SNR) is passed to $\sigma_{d_{L}(z)}$ via the Fisher matrix.

In the following we enter into the main part of this section where we describe the strain of GW interferometers.

Considering the transverse-traceless (TT) gauge, the strain $h(t)$ in the GW interferometers can be given by $[57,60]$

$$
h(t)=F_{+}(\theta, \phi, \psi) h_{+}(t)+F_{\times}(\theta, \phi, \psi) h_{\times}(t),
$$


where $F_{+}$and $F_{\times}$are the beam pattern functions of the Einstein Telescope; $\psi$ is the polarization angle; the angles $\theta$, $\phi$ effectively describe the location of the GW source with respect to the GW detector (here the Einstein Telescope); $h_{+}=h_{x x}=-h_{-y y}, h_{\times}=h_{x y}=h_{y x}$ [two independent components of the GW's tensor $h_{\mu \nu}$ in the transverse-traceless (TT) gauge]; see the details here [57]. Now, one can write down the antenna pattern functions of the Einstein Telescope as $[56,57,60]$

$$
\begin{aligned}
F_{+}^{(1)}(\theta, \phi, \psi)= & \frac{\sqrt{3}}{2}\left[\frac{1}{2}\left(1+\cos ^{2}(\theta)\right) \cos (2 \phi) \cos (2 \psi)\right. \\
& -\cos (\theta) \sin (2 \phi) \sin (2 \psi)], \\
F_{\times}^{(1)}(\theta, \phi, \psi)= & \frac{\sqrt{3}}{2}\left[\frac{1}{2}\left(1+\cos ^{2}(\theta)\right) \cos (2 \phi) \sin (2 \psi)\right. \\
& +\cos (\theta) \sin (2 \phi) \cos (2 \psi)] .
\end{aligned}
$$

For the remaining two interferometers, their antenna pattern functions can be derived using the above equations for $F_{+}^{(1)}(\theta, \phi, \psi)$ and $F_{\times}^{(1)}(\theta, \phi, \psi)$ and substituting $\phi$ by $\phi+120^{\circ}$ or $\phi+240^{\circ}$, because the three interferometers form an equilateral triangle, and hence, they make $60^{\circ}$ with each other.

Then, we follow the works of $[56,81]$ to derive the Fourier transform $\mathcal{H}(f)$ of the time domain waveform $h(t)$ considering the stationary phase approximation that leads to $\mathcal{H}(f)=\mathcal{A} f^{-7 / 6} \exp \left[i\left(2 \pi f t_{0}-\pi / 4+2 \psi(f / 2)-\varphi_{(2.0)}\right)\right]$, where $\mathcal{A}$ is the Fourier amplitude having the following expression

$$
\begin{aligned}
\mathcal{A}= & \frac{1}{d_{L}} \sqrt{F_{+}^{2}\left(1+\cos ^{2}(\omega)\right)^{2}+4 F_{\times}^{2} \cos ^{2}(\omega)} \\
& \times \sqrt{5 \pi / 96} \pi^{-7 / 6} \mathcal{M}_{c}^{5 / 6},
\end{aligned}
$$

in which $\mathcal{M}_{c}$ is dubbed as the "chirp mass," which is related to the total mass $M$ of the coalescing binary system $\left(M=m_{1}+m_{2}\right.$; here the component masses are $\left.m_{1}, m_{2}\right)$ and the symmetric mass ratio $\eta=m_{1} m_{2} / M^{2}$ by the relation $\mathcal{M}_{c}=M \eta^{3 / 5}$. Note that the masses here are actually the observed masses, which are related to the intrinsic masses as $M_{\mathrm{obs}}=(1+z) M_{\mathrm{int}}$, exhibiting an enhancement of a factor $(1+z)$.

Furthermore, in the expression for $\mathcal{A}$, the symbol $\omega$ denotes the angle of inclination of the binary's orbital angular momentum with the line of sight. Since the short gamma ray bursts (SGRBs) are usually expected to be strongly beamed, the coincidence observations of SGRBs suggest that the binaries should be aligned in such a way so that $\omega \simeq 0$ with its maximal inclination about $\omega=20^{\circ}$. Here, we make a comment that averaging the Fisher matrix over the inclination (i.e., $\omega$ ) and the polarization (i.e., $\psi$ ) under the constraint $\omega<90^{\circ}$ is almost (roughly) the same as setting $\omega=0$, considered in the simulation of [81]. Thus, during the simulation of the GW sources, one can safely consider $\omega=0$. However, during the estimation of the practical uncertainty of $d_{L}$, the uncertainty of inclination should be considered positively.

When the waveform of GW is known, one can calculate the signal-to-noise ratio (SNR). The SNR plays a very crucial role in detection of the GW event, because a GW detection is confirmed if the combined SNR of at least 8 is found in the Einstein Telescope [82,83] (see also $[56,57,59,84]$ for more details in this direction). In general, the combined SNR for the network employing three independent interferometers (just like in the Einstein Telescope) is $\rho=\sqrt{\sum_{i=1}^{3}\left(\rho^{(i)}\right)^{2}}$, where $\rho^{(i)}=$ $\sqrt{\left\langle\mathcal{H}^{(i)}, \mathcal{H}^{(i)}\right\rangle}$, and the inner product inside the square root follows $[56,57,60]$

$$
\langle a, b\rangle=4 \int_{f_{\text {lower }}}^{f_{\text {upper }}} \frac{\tilde{a}(f) \tilde{b}^{*}(f)+\tilde{a}^{*}(f) \tilde{b}(f)}{2} \frac{d f}{S_{h}(f)},
$$

where the sign " $\sim$ " placed over the symbols denotes their Fourier transformations and $S_{h}(f)$ is the one-side noise power spectral density, which for this article is taken to be the same as in [56].

Now, the instrumental error (following Fisher matrix approach) on $d_{L}$ can be estimated through the relation

$$
\sigma_{d_{L}}^{\text {inst }} \simeq \sqrt{\left\langle\frac{\partial \mathcal{H}}{\partial d_{L}}, \frac{\partial \mathcal{H}}{\partial d_{L}}\right\rangle^{-1}}
$$

Assuming that $d_{L}$ is independent of other parameters, and using the relation $\mathcal{H} \propto d_{L}^{-1}$, from (1), one can deduce that $\sigma_{d_{L}}^{\text {inst }} \simeq d_{L} / \rho$. Now, when we estimate the uncertainty of the measurement $d_{L}$, we should take into account the inclination $\omega$. At the same time we must consider the correlation between $d_{L}$ and $\omega$. While taking into account such correlation, the maximal effect of the inclination on the SNR, which is a factor of 2 (between $\omega=0$ and $\omega=90^{\circ}$ ), is considered. Now, in order to provide with an estimation of the ability of the GWSS to constrain the cosmological parameters, we double the estimation of the error imposed on the luminosity distance that goes as [81] $\sigma_{d_{L}}^{\text {inst }} \simeq \frac{2 d_{L}}{\rho}$. Moreover, under the short-wave approximation, GW are lensed in the same way as electromagnetic waves during propagation, resulting in an additional weak lensing error that is modeled as $\sigma_{d_{L}}^{\text {lens }}=0.05 z d_{L}$ in [57]. Consequently, the combined error is $\sigma_{d_{L}}=\sqrt{\left(\sigma_{d_{L}}^{\text {inst }}\right)^{2}+\left(\sigma_{d_{L}}^{\text {lens }}\right)^{2}}$, where the errors $\sigma_{d_{L}}^{\text {inst }}$ and $\sigma_{d_{L}}^{\text {lens }}$ are already defined above. 
Thus, following the method described above, one is now able to generate the future GWSS dataset consisting of $\left(z, d_{L}(z), \sigma_{d_{L}(z)}\right)$. As argued in [57], the constraining ability of Planck on cosmological parameters can only be reached with at least $1000 \mathrm{GW}$ events, corresponding to 10 years of observation by the Einstein Telescope; therefore, data of $1000 \mathrm{GW}$ events are mocked in this work.

Finally, we come to the last part of this section where we describe the approach to use the simulated GW data. The analysis with GW data is similar to the standard cosmological probes. For the GW standard siren measurements with $N$ simulated data points, the $\chi^{2}$ function is given by

$$
\chi_{\mathrm{GW}}^{2}=\sum_{i=1}^{N}\left[\frac{\bar{d}_{L}^{i}-d_{L}\left(\bar{z}_{i} ; \vec{\Theta}\right)}{\bar{\sigma}_{d_{L}}^{i}}\right]^{2},
$$

where $\bar{z}_{i}, \bar{d}_{L}^{i}$, and $\bar{\sigma}_{d_{L}}^{i}$ are respectively the $i$ th redshift, luminosity distance at this redshift, and the error of the luminosity distance of the simulated GW data for this particular redshift. Here, $\vec{\Theta}$ represents the set of cosmological parameters that we need to constrain.

We conclude this section with the following remark, which we believe to be important in the context of simulating GW data. We notice that different models of GW sources have been proposed in earlier investigations, e.g., [85-87], and the types or distributions of GW sources may vary from model to model. Whereas, for the purpose of this paper, what really matters is their impact on the observables, especially the error of luminosity distance. A detailed investigation regarding the merger of astrophysical binary systems is reported in Ref. [85], where the exact expressions for NS/BH merger rates are derived based on the physical process of star formation, and the current abundances of binary systems are normalized by the constraint from observational data $[88,89]$. For the sake of cross-check, we have also conducted a simulation following their approach and assume the same form of lensing error (this part is not considered in [85]). It turns out that the resulting $\delta d_{\mathrm{L}}$ of these two methods are quite close, as is shown in Fig. 1. And this is partly because the contribution of gravitational lensing (green line of Fig. 1) takes majority in the whole error budget (yellow and blue lines of Fig. 1), which means $\sigma_{d_{\mathrm{L}}}^{\text {lens }}>\sigma_{d_{\mathrm{L}}}^{\text {inst }}$. Thus, the validity of our approach is confirmed.

\section{STANDARD COSMOLOGICAL PROBES AND THE TOTAL LIKELIHOOD ANALYSIS INCLUDING GW}

Here we summarize the standard observational data used to analyze the models. In the following we outline a brief description for each dataset.

(1) CMB data: The cosmic microwave background radiation is an important cosmological data to

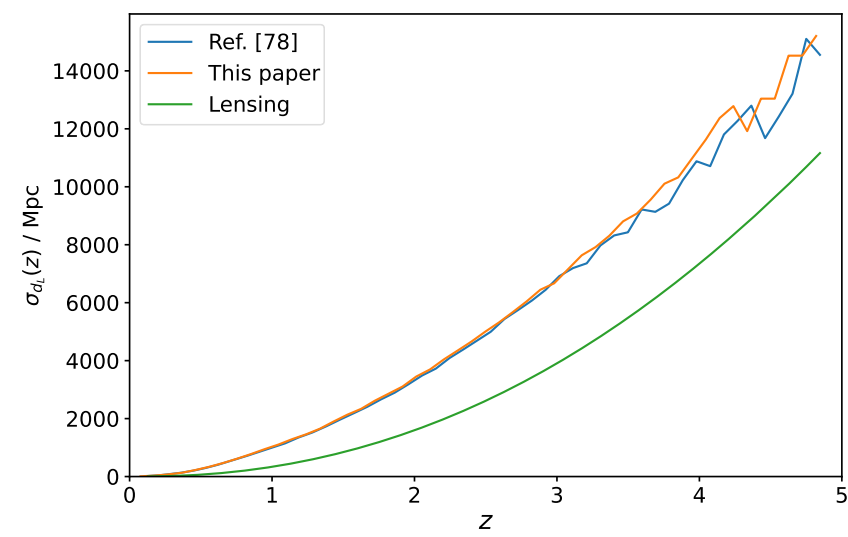

FIG. 1. Error of luminosity distance based on two sets of simulations. It is clear from the figure that the results of these two methods are quite close. In the plot we also show the contribution of gravitational lensing, which takes majority in the whole error budget, i.e., $\sigma_{d_{\mathrm{L}}}^{\text {lens }}>\sigma_{d_{\mathrm{L}}}^{\text {inst }}$.

analyze the dark energy models. In particular, we use the Planck 2015 measurements [49,50] that include the high- and low- $\ell$ TT likelihoods in the mutiple range $2 \leq \ell \leq 2508$ as well as the highand low- $\ell$ polarization likelihoods. The entire dataset is identified as Planck TTTEEE + lowTEB.

(2) BAO data: In this work we employ four distinct BAO data measured by different observational surveys. Precisely, we take the (i) 6dF Galaxy Survey measurement at $z_{\mathrm{eff}}=0.106$ [51], (ii) the Main Galaxy Sample of Data Release 7 of Sloan Digital Sky Survey at $z_{\text {eff }}=0.15$ [52], (iii) the CMASS sample from the latest Data Release 12 (DR12) of the Baryon Oscillation Spectroscopic Survey (BOSS) at $z_{\text {eff }}=0.57$, and finally (iv) the LOWZ sample from BOSS DR12 at $z_{\text {eff }}=0.32$ [53].

(3) Supernovae type Ia: The joint light curve sample [54] from SNIa data scattered in the redshift region $z \in[0.01,1.30]$ has been considered. The total number of SNIa in this region is 740 .

(4) Cosmic chronometers: We also add the Hubble parameter measurements from the cosmic chronometers. The cosmic chronometers are the most massive and passively evolving galaxies. The measurements of the Hubble parameters from the cosmic chronometers are promising to estimate the cosmological parameters; see [55]. The total number of Hubble data points we consider in this analysis is thirty distributed in the redshift region $0<z<2$.

Now in order to extract the observational constraints on the proposed dynamical dark energyparametrizations for several combinations of the cosmological datasets, we use an efficient package, namely, the Markov chain Monte Carlo package cosmomc $[90,91]$ which is equipped with the well-known convergence statistic by GelmanRubin [92]. The cosmomc package also includes the 
TABLE I. The flat priors on various cosmological parameters used for constraining the dynamical dark energy models.

\begin{tabular}{lc}
\hline \hline Parameter & Prior \\
\hline$\Omega_{\mathrm{b}} h^{2}$ & {$[0.005,0.1]$} \\
$\Omega_{\mathrm{c}} h^{2}$ & {$[0.01,0.99]$} \\
$\tau$ & {$[0.01,0.8]$} \\
$n_{s}$ & {$[0.5,1.5]$} \\
$\log \left[10^{10} A_{s}\right]$ & {$[2.4,4]$} \\
$100 \theta_{M C}$ & {$[0.5,10]$} \\
$w_{0}$ & {$[-2,0]$} \\
$w_{a}$ & {$[-3,3]$} \\
\hline \hline
\end{tabular}

support for Planck 2015 likelihood code [50]. One can avail this code online [93], and it is freely available. The parameter space that we constrain in this work is as follows:

$$
\mathcal{P} \equiv\left\{\Omega_{b} h^{2}, \Omega_{c} h^{2}, 100 \theta_{M C}, \tau, n_{s}, \log \left[10^{10} A_{s}\right], w_{0}, w_{a}\right\},
$$

where $\Omega_{b} h^{2}, \Omega_{c} h^{2}$ are respectively the physical density for baryons and cold dark matter; $\theta_{M C}$ is the ratio of sound horizon to the angular diameter distance; $\tau$ refers to the reionization optical depth; $n_{s}$ is the scalar spectral index; $A_{s}$ is the amplitude of the primordial scalar power spectrum; $w_{0}, w_{a}$ are the key parameters of all the dark energy parametrizations. In Table I we describe the flat priors on the cosmological parameters used during the analysis of the models. (PRIORS ON LOG: $w_{a}$ : Fix it)

\section{RESULTS AND ANALYSIS}

Let us now summarize the main observational results extracted from the dynamical dark energy models (10)-(13) after the inclusion of the simulated $\mathrm{GW}$ data. In the following we describe the results for each model in detail.

\section{A. CPL parametrization}

First of all, we have constrained the CPL parametrization of Eq. (10) using the standard cosmological probes, such as CMB, BAO, JLA, and CC (summarized in the upper half of Table II), and then using the best-fit values of the model parameters of this model, we have generated the GW catalogue comprising 1000 simulated GW events. So, here we have considered CPL as the fiducial model. In Fig. 2, we have shown the luminosity distance $d_{L}(z)$ versus $z$ graphics for the 1000 simulated GW events. Now, incorporating the simulated GW events with the standard cosmological probes, we have constrained the CPL parametrization. The summary of the observational constraints on the CPL model after the inclusion of the simulated GW data is shown in the lower half of Table II.

In Fig. 3 we present the comparisons between the constraining results of the datasets before and after the inclusion of the $\mathrm{GW}$ data to the standard cosmological probes mentioned above, where, in particular, we show the one-dimensional (1D) marginalized posterior distributions for some selected parameters of the model as well as the two-dimensional (2D) contour plots between several combinations of the model parameters of this parametrization. Specifically, the upper left panel of Fig. 3 presents the comparisons between the datasets $\mathrm{CMB}$ and $\mathrm{CMB}+\mathrm{GW}$; the upper right panel of Fig. 3 is for $\mathrm{CMB}+\mathrm{BAO}$ and $\mathrm{CMB}+\mathrm{BAO}+\mathrm{GW}$; the lower left panel of Fig. 3 is for $\mathrm{CMB}+\mathrm{BAO}+\mathrm{JLA}$ and $\mathrm{CMB}+\mathrm{BAO}+\mathrm{JLA}+\mathrm{GW}$; finally the lower right panel of Fig. 3 is for $\mathrm{CMB}+\mathrm{BAO}+$ $\mathrm{JLA}+\mathrm{CC}$ and $\mathrm{CMB}+\mathrm{BAO}+\mathrm{JLA}+\mathrm{CC}+\mathrm{GW}$. In the following we describe the effects of $\mathrm{GW}$ on the model parameters corresponding to different observational datasets.

In the second column of Table II, we present the observational constraints on the model parameters for the datasets $\mathrm{CMB}$ and $\mathrm{CMB}+\mathrm{GW}$. One can clearly notice that the inclusion of GW to $\mathrm{CMB}$ is effective to reduce the error bars on some of the parameter space of this model; see the top left panel of Fig. 3 for a better view on the parameter space. In particular, one can note the significant improvement in the estimations of the Hubble constant as follows: $H_{0}=83.06_{-7.98}^{+15.10}$ (68\% C.L., CMB) and $H_{0}=80.75_{-1.92}^{+1.71}$ $(68 \%$ C.L., CMB + GW). We note that due to the inclusion of GW to CMB, the error bars on $H_{0}$ are reduced by several factors. We also note that the matter density parameter at present, $\Omega_{m 0}$, for CMB alone is constrained to be small compared to Planck's estimation [74] and the inclusion of GW to CMB again improves the parameter space, but slightly (see the lower half of Table II). However, significant improvement is found in the estimation of $\sigma_{8}$, where one can notice that the inclusion of $\mathrm{GW}$ to $\mathrm{CMB}$ reduces the error bars by several factors. Concerning the two key parameters of this model, namely, $w_{0}$ and $w_{a}$, the effects of GW to $\mathrm{CMB}$ are quite evident. The addition of GW to $\mathrm{CMB}$ significantly improves the parameter space by reducing the error bars: $w_{0}=-1.218_{-0.597}^{+0.302}$ (68\% C.L., $\mathrm{CMB})$ and $w_{0}=-1.168_{-0.212}^{+0.180}$ (68\% C.L., CMB + GW). Although the deviation in the mean value of $w_{0}$, defined by $\left|\Delta w_{0}\right|=\left|w_{0}(\mathrm{CMB})-w_{0}(\mathrm{CMB}+\mathrm{GW})\right|=0.05$, is very small, the effective nature of $\mathrm{GW}$ is visible through its constraining power in terms of the reduction of the error bars on $w_{0}$. Overall, the inclusion of GW to CMB shifts $w_{0}$ towards -1 boundary, although its phantom nature is still allowed within $68 \%$ C.L. The constraints on $w_{a}$ for CMB alone are not stringent (the upper limit is $w_{a}<0.526$ at 95\% C.L.), but the inclusion of $\mathrm{GW}$ again reduces its error bars with $w_{a}=-1.081_{-0.640}^{+0.842}(68 \%$ C.L., CMB + GW). In fact, the power of GW is clear from both the 1D posterior distributions of some parameters as well as the 2D contour plots shown in the top left panel of Fig. 3. From this figure (top left panel of Fig. 3), one can clearly understand that a significant improvement in the parameter space is due to the inclusion of GW to CMB. 
We now present the cosmological constraints from $\mathrm{CMB}+\mathrm{BAO}$ and $\mathrm{CMB}+\mathrm{BAO}+\mathrm{GW}$. With these, we could be able to see how GW data affect this particular combination. The summary of the observational constraints is shown in the third column of Table II and the corresponding graphical variations are shown in the top right panel of Fig. 3. From the table, one can see that the inclusion of $\mathrm{BAO}$ to both $\mathrm{CMB}$ and $\mathrm{CMB}+\mathrm{GW}$ lowers $H_{0}$ returning similar mean values as follows: $H_{0}=$ $64.36_{-3.23}^{+2.05}(68 \%, \mathrm{CMB}+\mathrm{BAO})$ and $H_{0}=63.77_{-1.52}^{+1.37}$ $(68 \%, \mathrm{CMB}+\mathrm{BAO}+\mathrm{GW})$. The error bars on $H_{0}$ are reduced after the inclusion of GW data. One can also notice that for both the analyses, $w_{0}$ allows very higher values and $w_{a}$ takes very lower values, exactly the same as recently found in [94]. The interesting fact is that, after the inclusion of $\mathrm{BAO}$ to $\mathrm{CMB}$, all the parameters are correlated with each other (see the top right panel of 3), and this remains true even after the inclusion of $\mathrm{GW}$ to the combined analysis $\mathrm{CMB}+\mathrm{BAO}$. But, indeed, it is quite clear that the dataset $\mathrm{CMB}+\mathrm{BAO}+\mathrm{GW}$ provides better constraints than $\mathrm{CMB}+\mathrm{BAO}$.

We now discuss the cosmological constraints in the presence of the JLA data to the previous datasets, which means precisely we discuss the constraints from $\mathrm{CMB}+$ $\mathrm{BAO}+\mathrm{JLA}$ and $\mathrm{CMB}+\mathrm{BAO}+\mathrm{JLA}+\mathrm{GW}$. The summary of the observational constraints is shown in the fourth column of Table II and the graphical distributions are shown in the bottom left panel of Fig. 3. From this analysis, it is again clear that the inclusion of $\mathrm{GW}$ data reduces the error bars on all the parameters. In particular, one can see the $68 \%$ C.L. constraints on the Hubble constant as $H_{0}=67.94_{-1.08}^{+1.09}(\mathrm{CMB}+\mathrm{BAO}+\mathrm{JLA}), H_{0}=66.98_{-0.55}^{+0.55}$ $(\mathrm{CMB}+\mathrm{BAO}+\mathrm{JLA}+\mathrm{GW})$, which show that the inclusion of GW shifts $H_{0}$ towards its lower values and the error bars are reduced by a factor of 2 . Concerning the two key parameters of this model, which means $w_{0}$ and $w_{a}$, we have some interesting observations. We see that for both the combinations, $w_{0}$ approaches near the -1 border with

TABLE II. Sixty-eight percent and 95\% C.L. constraints on the Chevallier-Polarski-Linder parametrization (10) using various combinations of the observational data with and without the GW data. The upper panel represents the constraints on the model without the GW data while in the lower panel we present the corresponding constraints using the GW data. For the CMB only case the upper limits of the $w_{a}$ parameter at $68 \%$ and $95 \%$ C.L. are reported. Here, $\Omega_{m 0}$ is the present value of $\Omega_{m}=\Omega_{b}+\Omega_{c}$ and $H_{0}$ is in units of $\mathrm{km} \mathrm{s}^{-1} \mathrm{Mpc}^{-1}$.

\begin{tabular}{|c|c|c|c|c|}
\hline Parameters & $\mathrm{CMB}$ & $\mathrm{CMB}+\mathrm{BAO}$ & $\mathrm{CMB}+\mathrm{BAO}+\mathrm{JLA}$ & $\mathrm{CMB}+\mathrm{BAO}+\mathrm{JLA}+\mathrm{CC}$ \\
\hline$\Omega_{c} h^{2}$ & $0.1190_{-0.0014-0.0027}^{+0.0014+0.0027}$ & $0.1191_{-0.0013-0.0027}^{+0.0014+0.0026}$ & $0.1191_{-0.0013-0.0026}^{+0.0013+0.0025}$ & $0.1190_{-0.0013-0.0025}^{+0.0013+0.0024}$ \\
\hline$\Omega_{b} h^{2}$ & $0.02228_{-0.00016-0.00031}^{+0.00015+0.00031}$ & $0.02226_{-0.00015-0.00029}^{+0.00015+0.00029}$ & $0.02226_{-0.00014-0.00030}^{+0.00014+0.00030}$ & $0.02228_{-0.00016-0.00029}^{+0.00014+0.00030}$ \\
\hline $100 \theta_{M C}$ & $1.04081_{-0.00032-0.00064}^{+0.00032+0.00062}$ & $1.04078_{-0.00032-0.00064}^{+0.00033+0.00063}$ & $1.04079_{-0.00032-0.00063}^{+0.00032+0.00063}$ & $1.04081_{-0.00032-0.00063}^{+0.00033+0.00063}$ \\
\hline$\tau$ & \multicolumn{2}{|r|}{$0.078_{-0.017-0.034}^{+0.017+0.034}$} & $0.080_{-0.017-0.034}^{+0.017+0.034}$ & $0.081_{-0.017-0.034}^{+0.017+0.033}$ \\
\hline$n_{s}$ & $0.9667_{-0.0044-0.0087}^{+0.0044+0.0089}$ & $0.9665_{-0.0044-0.0084}^{+0.0044+0.0091}$ & $0.9666_{-0.0044-0.0089}^{+0.0045+0.0088}$ & $0.9665_{-0.0043-0.0082}^{+0.0043+0.0085}$ \\
\hline $\ln \left(10^{10} A_{s}\right)$ & $3.083_{-0.034-0.068}^{+0.035+0.066}$ & $3.090_{-0.033-0.066}^{+0.034+0.066}$ & $3.092_{-0.033-0.067}^{+0.033+0.066}$ & $3.094_{-0.033-0.065}^{+0.033+0.066}$ \\
\hline$w_{0}$ & $-1.218_{-0.597-0.782}^{+0.302+0.856}$ & $-0.524_{-0.236-0.514}^{+0.374+0.524}$ & $-0.909_{-0.123-0.201}^{+0.095+0.216}$ & $-0.909_{-0.116-0.209}^{+0.099+0.213}$ \\
\hline$w_{a}$ & $<-0.446<0.526$ & $-1.403_{-1.021-1.466}^{+0.731+1.570}$ & $-0.409_{-0.277-0.777}^{+0.517+0.689}$ & $-0.399_{-0.297-0.724}^{+0.423+0.676}$ \\
\hline$\Omega_{m 0}$ & $0.218_{-0.081-0.097}^{+0.028+0.146}$ & $0.344_{-0.026-0.054}^{+0.032+0.051}$ & $0.308_{-0.011-0.019}^{+0.009+0.020}$ & $0.308_{-0.011-0.019}^{+0.010+0.020}$ \\
\hline$\sigma_{8}$ & $0.960_{-0.065-0.185}^{+0.118+0.152}$ & $0.803_{-0.030-0.051}^{+0.024+0.053}$ & $0.835_{-0.017-0.035}^{+0.018+0.035}$ & $0.835_{-0.017-0.033}^{+0.017+0.034}$ \\
\hline$H_{0}$ & $83.06_{-7.98-21.61}^{+15.10+18.40}$ & $64.36_{-3.23-4.67}^{+2.05+5.26}$ & $67.94_{-1.08-2.05}^{+1.09+2.10}$ & $67.92_{-1.09-2.10}^{+1.09+2.14}$ \\
\hline Parameters & $\mathrm{CMB}+\mathrm{GW}$ & $\mathrm{CMB}+\mathrm{BAO}+\mathrm{GW}$ & $\mathrm{B}+\mathrm{BAO}+\mathrm{JLA}+\mathrm{GW}$ & $\mathrm{CMB}+\mathrm{BAO}+\mathrm{JLA}+\mathrm{CC}+\mathrm{GW}$ \\
\hline$\Omega_{c} h^{2}$ & $0.1186_{-0.0012-0.0024}^{+0.0012+0.0024}$ & $0.1188_{-0.0013-0.0025}^{+0.0013+0.0025}$ & $0.1189_{-0.0012-0.0023}^{+0.0012+0.0024}$ & $0.1188_{-0.0013-0.0025}^{+0.0013+0.0025}$ \\
\hline$\Omega_{b} h^{2}$ & $0.02233_{-0.00014-0.00027}^{+0.00014+0.00028}$ & $0.02231_{-0.00015-0.00030}^{+0.00015+0.00028}$ & $0.02226_{-0.00016-0.00030}^{+0.00015+0.00030}$ & $0.02231_{-0.00015-0.00029}^{+0.00015+0.00029}$ \\
\hline $100 \theta_{M C}$ & $1.04088_{-0.00030-0.00062}^{+0.00031+0.0060}$ & $1.04088_{-0.00032-0.00063}^{+0.00032+0.00062}$ & $1.04079_{-0.00032-0.00063}^{+0.00032+0.00061}$ & $1.04088_{-0.00030-0.00061}^{+0.00031+0.00061}$ \\
\hline$\tau$ & $0.079_{-0.017-0.033}^{+0.017+0.033}$ & $0.081_{-0.017-0.033}^{+0.017+0.034}$ & $0.081_{-0.017-0.0315}^{+0.017+0.034}$ & $0.082_{-0.017-0.034}^{+0.018+0.034}$ \\
\hline$n_{s}$ & $0.9677_{-0.0042-0.0081}^{+0.0041+0.0082}$ & $0.9675_{-0.0043-0.0087}^{+0.0043+0.0086}$ & $0.9670_{-0.0041-0.0082}^{+0.0041+0.0078}$ & $0.9675_{-0.0042-0.0086}^{+0.0043+0.0086}$ \\
\hline $\ln \left(10^{10} A_{s}\right)$ & $3.089_{-0.033-0.067}^{+0.034+0.064}$ & $3.093_{-0.033-0.064}^{+0.034+0.065}$ & $3.094_{-0.033-0.062}^{+0.033+0.067}$ & $3.096_{-0.034-0.066}^{+0.035+0.066}$ \\
\hline$w_{0}$ & $-1.168_{-0.212-0.361}^{+0.180+0.385}$ & $-0.465_{-0.200-0.360}^{+0.189+0.359}$ & $-0.904_{-0.080-0.144}^{+0.070+0.155}$ & $-0.902_{-0.062-0.124}^{+0.064+0.124}$ \\
\hline$w_{a}$ & $-1.081_{-0.640-1.558}^{+0.842+1.303}$ & $-1.523_{-0.562-1.160}^{+0.642+1.071}$ & $-0.256_{-0.227-0.523}^{+0.263+0.549}$ & $-0.373_{-0.226-0.500}^{+0.263+0.451}$ \\
\hline$\Omega_{m 0}$ & $0.218_{-0.010-0.019}^{+0.010+0.020}$ & $0.349_{-0.016-0.031}^{+0.017+0.031}$ & $0.318_{-0.006-0.012}^{+0.006+0.012}$ & $0.309_{-0.004-0.009}^{+0.004+0.009}$ \\
\hline$\sigma_{8}$ & $0.945_{-0.022-0.040}^{+0.020+0.043}$ & $\begin{array}{c}0.797_{-0.019-0.033}^{+0.017+0.036} \\
-0.03\end{array}$ & $0.822_{-0.015-0.027}^{+0.015+0.029}$ & $0.831_{-0.015-0.029}^{+0.015+0.029}$ \\
\hline$H_{0}$ & $\begin{array}{l}80.75_{-1.92-3.37}^{+1.71+3.68} \\
\end{array}$ & $\begin{array}{r}63.77_{-1.52-2.77}^{+1.37+2.80} \\
\end{array}$ & $66.98_{-0.55-1.10}^{+0.55+1.12}$ & $\begin{array}{c}67.72_{-0.35-0.71}^{+0.36+0.71} \\
\end{array}$ \\
\hline
\end{tabular}



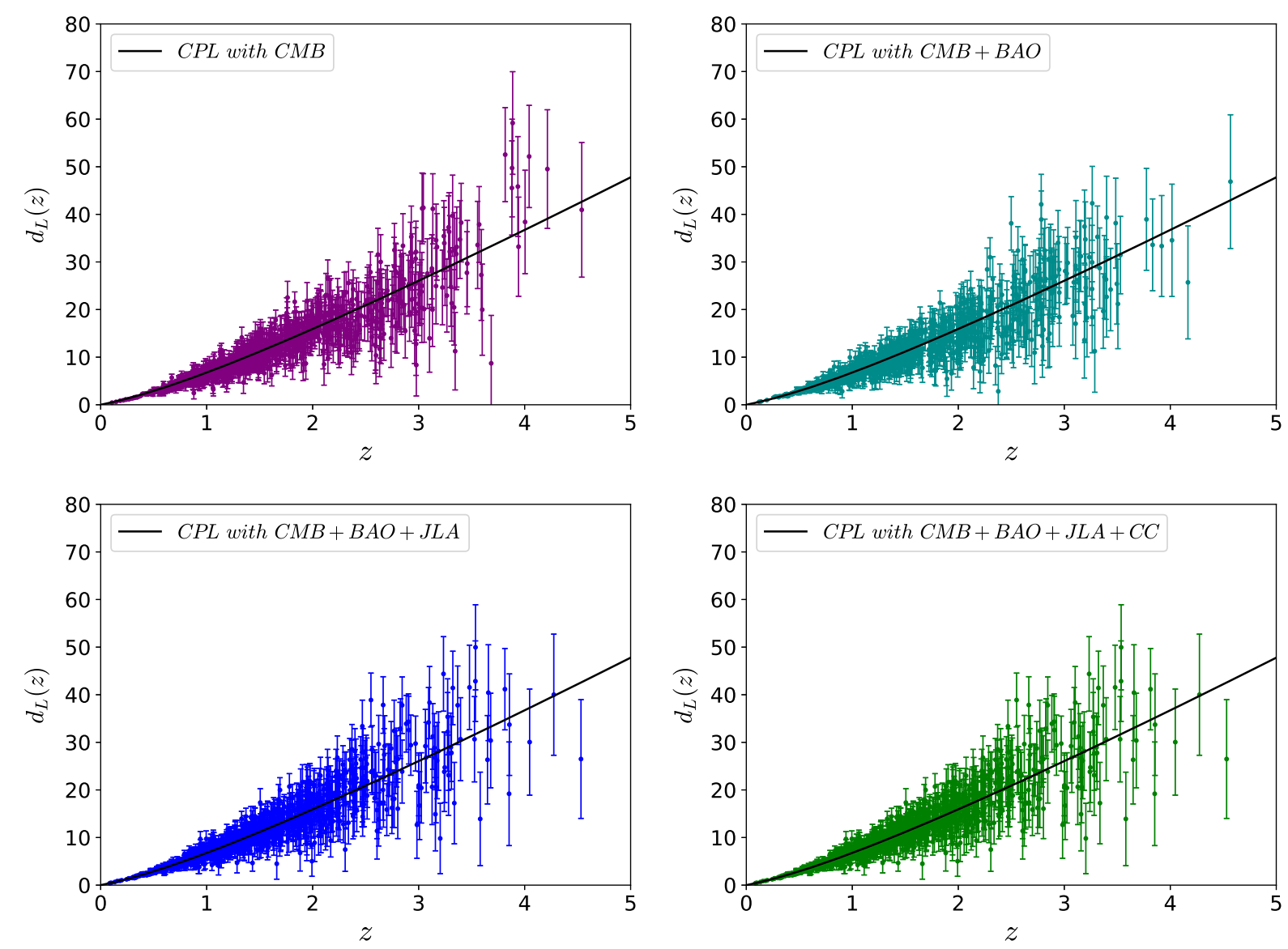

FIG. 2. For the fiducial CPL model, we first constrain the cosmological parameters using the datasets $\mathrm{CMB}$, CMB $+\mathrm{BAO}$, $\mathrm{CMB}+\mathrm{BAO}+\mathrm{JLA}$, and $\mathrm{CMB}+\mathrm{BAO}+\mathrm{JLA}+\mathrm{CC}$ and then we use the best-fit values of the parameters for "each dataset" to generate the corresponding GW catalogue. Following this, in each panel we show $d_{L}(z)$ vs $z$ catalogue with the corresponding error bars for 1000 simulated GW events. The upper left and upper right panels respectively present the catalogue $\left(z, d_{L}(z)\right)$ with the corresponding error bars for 1000 simulated events derived using the CMB alone and CMB + BAO dataset. The lower left and lower right panels respectively present the catalogue $\left(z, d_{L}(z)\right)$ with the corresponding error bars for 1000 simulated events derived using the $\mathrm{CMB}+\mathrm{BAO}+\mathrm{JLA}$ and $\mathrm{CMB}+\mathrm{BAO}+\mathrm{JLA}+\mathrm{CC}$ datasets.

$w_{0}=-0.909_{-0.123}^{+0.095}(68 \%$ C.L., CMB $+\mathrm{BAO}+\mathrm{JLA})$ and $w_{0}=-0.904_{-0.080}^{+0.070}(68 \%$ C.L., $\quad$ CMB $+\mathrm{BAO}+\mathrm{JLA}+$ $\mathrm{GW})$. From the highest peak of the 1D posterior distributions of $w_{0}$ (see the bottom left panel of Fig. 3) for both the datasets, $w_{0}>-1$ is strongly supported while the tails of the posterior distributions of this parameter are lying from quintessence to the phantom regime due to the error bars on $w_{0}$. The improvement in $w_{a}$ is also transparent: $w_{a}=-0.409_{-0.277}^{+0.517}(68 \%$ C.L., CMB + BAO + JLA $)$ and $w_{a}=-0.256_{-0.227}^{+0.263} \quad(68 \%$ C.L., $\mathrm{CMB}+\mathrm{BAO}+\mathrm{JLA}+$ $\mathrm{GW})$. So, from both the observational datasets, dynamical nature is allowed while one can also note that $w_{a}=0$ is also not excluded in $68 \%$ C.L. Finally, we mention the correlations between the parameters clearly shown in the bottom left panel of Fig. 3, where we see that such correlations are not affected by the GW data. However, we mention that the inclusion of JLA decreases the correlation between some of the combinations of the parameters. And, in particular, we find that some of parameters are uncorrelated; for instance, we see that $\sigma_{8}$ seems to be uncorrelated with $w_{0}$ and $w_{a}$.

We now discuss the last two analyses for this model, namely, with $\mathrm{CMB}+\mathrm{BAO}+\mathrm{JLA}+\mathrm{CC}$ and its companion $\mathrm{CMB}+\mathrm{BAO}+\mathrm{JLA}+\mathrm{CC}+\mathrm{GW}$. The summary of the observational constraints is shown in the last column of Table II and in the bottom right panel of Fig. 3 we compare these datasets. From the analysis, we clearly notice that the inclusion of the GW data improves the parameter space in an effective way. In fact, the maximum effects are seen in $H_{0}$ and $\Omega_{m 0}$ (see the $1 \mathrm{D}$ posterior distributions of these parameters as well). In particular, one can look at the improvements of the Hubble parameter after the inclusion of GW data: $H_{0}=67.92_{-1.09}^{+1.09}$ $(68 \%, \mathrm{CMB}+\mathrm{BAO}+\mathrm{JLA}+\mathrm{CC})$ and $H_{0}=67.72_{-0.35}^{+0.36}$ (68\% C.L., CMB + BAO+JLA + CC + GW). Furthermore, the estimations of other parameters can also be visualized in a similar fashion. Concerning the key parameters of this parametrization, namely, $\left(w_{0}, w_{a}\right)$, we observe 

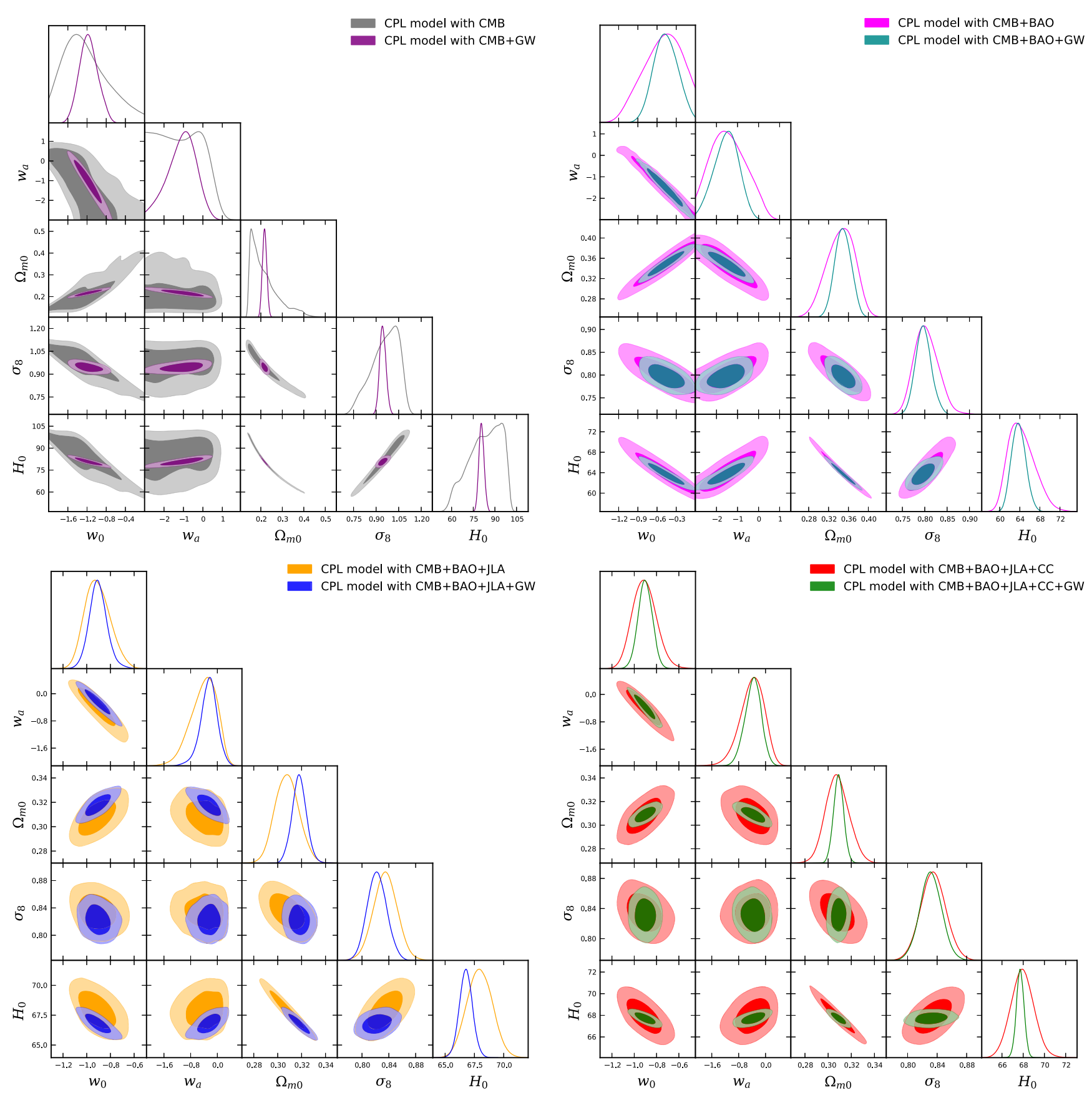

FIG. 3. Sixty-eight percent and 95\% C.L. contour plots for various combinations of some selected parameters of the CPL model (10) using different observational data in the presence (absence) of the GW data.

significant changes on their constraints. Looking at the $68 \%$ C.L. constraints on $w_{0}$ where $w_{0}=-0.909_{-0.116}^{+0.099}$ $(\mathrm{CMB}+\mathrm{BAO}+\mathrm{JLA}+\mathrm{CC})$ and $w_{0}=-0.902_{-0.062}^{+0.064}$ $(\mathrm{CMB}+\mathrm{BAO}+\mathrm{JLA}+\mathrm{CC}+\mathrm{GW})$, one can see that after the inclusion of $\mathrm{GW}$ at $68 \%$ upper $\mathrm{CL}$ error bars on $w_{0}$ are reduced by a factor of 2 . For the other parameter $w_{a}: w_{a}=-0.399_{-0.297}^{+0.423} \quad(68 \% \quad$ C.L., $\quad \mathrm{CMB}+\mathrm{BAO}+$ $\mathrm{JLA}+\mathrm{CC})$ and $w_{a}=-0.373_{-0.226}^{+0.263}(68 \%$ C.L., CMB + $\mathrm{BAO}+\mathrm{JLA}+\mathrm{CC}+\mathrm{GW})$, although the reduction of the error bars is not very significant compared to $w_{0}$; however, such improvements are clearly visualized. Moreover, looking at the constraints on $w_{0}$, one can also argue that for both the datasets, the dark energy equation of state at present exhibits its quintessential nature (i.e., $w_{0}>-1$ ). This feature is actually clear if one looks at the highest peaks of the 1D posterior distributions of $w_{0}$ in Fig. 3 (see the bottom right panel of this figure). Additionally, we find that for the final combination, that means for $\mathrm{CMB}+\mathrm{BAO}+$ $\mathrm{JLA}+\mathrm{CC}+\mathrm{GW}$, within $68 \%$ C.L., $w_{a} \neq 0$. It means that 

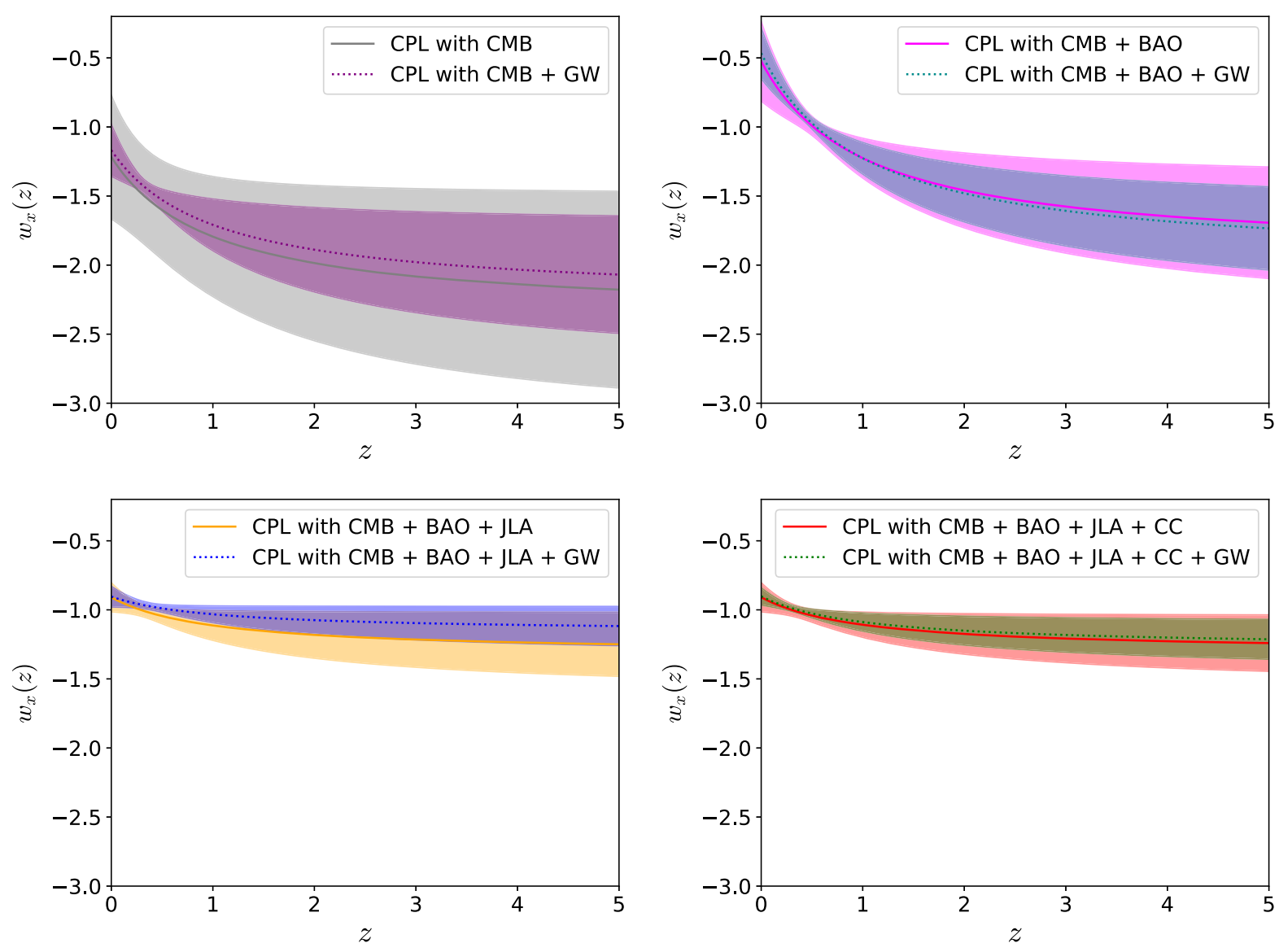

FIG. 4. The evolution of the dark energy equation of state for the CPL parametrziation is shown for different datasets taking the mean values of the key parameters $w_{0}$ and $w_{a}$ from the corresponding analysis with and without the GW data. The solid curves stand for the evolution of $w_{x}(z)$ for the standard cosmological probes while the dotted curves stand for the dataset in the presence of the GW data. The shaded regions show the $68 \%$ C.L. constraints on these two parameters.

a dynamical character is allowed within this confidence level. Concerning the correlations between the parameters, one may draw similar conclusions as found in the previous two datasets, namely, $\mathrm{CMB}+\mathrm{BAO}+\mathrm{JLA}$ and $\mathrm{CMB}+$ $\mathrm{BAO}+\mathrm{JLA}+\mathrm{GW}$.

Finally, using the mean values of $\left(w_{0}, w_{a}\right)$ from all the datasets, in Fig. 4 we have shown the qualitative evolution of the dark energy equation of state $w_{x}(z)$ for this model. The solid lines in each plot stand for the $w_{x}(z)$ curve for the usual cosmological probe and the dotted lines depict the evolution of $w_{x}(z)$ in the presence of the GW data. In each plot the shaded regions (with similar colors to the corresponding curves) present the $68 \%$ regions for the parameters $w_{0}, w_{a}$ corresponding to each dataset (with or without the GW data). From this figure (i.e., Fig. 4) one can see the addition of GW to the standard cosmological data certainly improves the parameter space. The maximum effects of GW are visible with the CMB data alone.

\section{B. Logarithmic parametrization}

In a similar fashion, we constrain the logarithmic parametrization (11) using the standard cosmological probes, such as CMB, BAO, JLA, and CC (summarized in the upper half of Table III), and then using the best-fit values of the model parameters, we have generated the GW catalogue comprising 1000 simulated GW events. In Fig. 5, we have shown the relation $d_{L}(z)$ vs $z$ for the 1000 simulated GW events. Now, using the simulated GW events with the standard cosmological probes, we have constrained this parametrization. The summary of the observational constraints on the CPL model after the inclusion of the simulated GW data is shown in the lower half of Table III.

In Fig. 6 we show the 1D marginalized posterior distributions for some specific parameters of this model as well as the $2 \mathrm{D}$ contour plots considering several combinations of the model parameters. From a first look at the upper and lower halves of Table III, one could clearly see that the inclusion of GW data to the standard cosmological probes significantly improves the model parameters of this parametrization, a similar observation already found in CPL parametrization. Let us now describe how GW works with different observational datasets presented here.

We begin the analyses with $\mathrm{CMB}$ data alone and $\mathrm{CMB}+\mathrm{GW}$. The results of both the analyses are 
TABLE III. Sixty-eight percent and 95\% C.L. constraints on the logarithmic parametrization (11) using various combinations of the observational data with and without the GW data. The upper panel represents the constraints without the GW data while in the lower panel we present the corresponding constraints using the GW data. Here, $\Omega_{m 0}$ is the present value of $\Omega_{m}=\Omega_{b}+\Omega_{c}$ and $H_{0}$ is in units of $\mathrm{km} \mathrm{s}^{-1} \mathrm{Mpc}^{-1}$

\begin{tabular}{|c|c|c|c|c|}
\hline Parameters & CMB & $\mathrm{CMB}+\mathrm{BAO}$ & $\mathrm{CMB}+\mathrm{BAO}+\mathrm{JLA}$ & $\mathrm{CMB}+\mathrm{BAO}+\mathrm{JLA}+\mathrm{CC}$ \\
\hline$\overline{\Omega_{c} h^{2}}$ & $0.1190_{-0.0014-0.0027}^{+0.0014+0.0028}$ & $0.1193_{-0.0014-0.0026}^{+0.0013+0.0026}$ & $0.1193_{-0.0014-0.0025}^{+0.0013+0.0025}$ & $0.1192_{-0.0013-0.0025}^{+0.0012+0.0026}$ \\
\hline$\Omega_{b} h^{2}$ & $0.02229_{-0.00016-0.00031}^{+0.00016+0.00031}$ & $0.02225_{-0.00015-0.00030}^{+0.00015+0.00031}$ & $0.02226_{-0.00015-0.00029}^{+0.00015+0.00030}$ & $0.02226_{-0.00015-0.00030}^{+0.00015+0.00030}$ \\
\hline $100 \theta_{M C}$ & $1.04081_{-0.00032-0.00066}^{+0.00033+0.00065}$ & $1.04075_{-0.00032-0.00065}^{+0.00032+0.00065}$ & $1.04075_{-0.00033-0.00065}^{+0.00032+0.0006}$ & $1.04078_{-0.00032-0.00065}^{+0.00031+0.00059}$ \\
\hline$\tau$ & $0.074_{-0.017-0.034}^{+0.017+0.034}$ & $0.076_{-0.017-0.034}^{+0.018+0.033}$ & $0.078_{-0.017-0.033}^{+0.017+0.034}$ & $0.079_{-0.017-0.034}^{+0.018+0.033}$ \\
\hline$n_{s}$ & $0.9668_{-0.0045-0.0090}^{+0.0045+0.0087}$ & $0.9659_{-0.0045-0.0087}^{+0.0045+0.0089}$ & $0.9661_{-0.0044-0.0085}^{+0.0043+0.0088}$ & $0.9663_{-0.0042-0.0085}^{+0.0043+0.0087}$ \\
\hline $\ln \left(10^{10} A_{s}\right)$ & $3.081_{-0.034-0.067}^{+0.034+0.067}$ & $3.085_{-0.034-0.068}^{+0.035+0.065}$ & $3.089_{-0.0335-0.0671}^{+0.0334+0.0646}$ & $3.091_{-0.034-0.066}^{+0.034+0.065}$ \\
\hline$w_{0}$ & $-1.058_{-0.550-0.759}^{+0.354+0.865}$ & $-0.429_{-0.223-0.386}^{+0.265+0.429}$ & $-0.895_{-0.098-0.169}^{+0.084+0.177}$ & $-0.894_{-0.097-0.158}^{+0.072+0.166}$ \\
\hline$w_{a}$ & $-1.579_{-1.421-1.421}^{+1.579+1.579}$ & $-1.301_{-0.570-0.967}^{+0.549+0.979}$ & $-0.365_{-0.083-0.450}^{+0.365+0.365}$ & $-0.352_{-0.137-0.416}^{+0.293+0.352}$ \\
\hline$\Omega_{m 0}$ & $0.219_{-0.082-0.097}^{+0.030+0.136}$ & $0.356_{-0.024-0.047}^{+0.026+0.043}$ & $0.308_{-0.011-0.021}^{+0.010+0.021}$ & $0.309_{-0.010-0.019}^{+0.010+0.020}$ \\
\hline$\sigma_{8}$ & $0.959_{-0.067-0.176}^{+0.122+0.152}$ & $0.795_{-0.026-0.044}^{+0.023+0.048}$ & $0.835_{-0.018-0.034}^{+0.017+0.034}$ & $0.835_{-0.017-0.035}^{+0.017+0.033}$ \\
\hline$H_{0}$ & $82.78_{-8.34-20.63}^{+15.48+18.54}$ & $63.30_{-2.52-4.02}^{+1.87+4.32}$ & $67.93_{-1.19-2.18}^{+1.11+2.22}$ & $67.84_{-1.14-2.01}^{+1.05+2.13}$ \\
\hline Parameters & $\mathrm{CMB}+\mathrm{GW}$ & $\mathrm{CMB}+\mathrm{BAO}+\mathrm{GW}$ & $\mathrm{CMB}+\mathrm{BAO}+\mathrm{JLA}+\mathrm{GW}$ & $\mathrm{CMB}+\mathrm{BAO}+\mathrm{JLA}+\mathrm{CC}+\mathrm{GW}$ \\
\hline$\Omega_{c} h^{2}$ & $0.1179_{-0.0012-0.0023}^{+0.0012+0.0023}$ & $0.1192_{-0.0013-0.0027}^{+0.0013+0.0026}$ & $0.1194_{-0.0013-0.0025}^{+0.0012+0.0026}$ & $0.1192_{-0.0012-0.0023}^{+0.0012+0.0024}$ \\
\hline$\Omega_{b} h^{2}$ & $0.02241_{-0.00014-0.00027}^{+0.00013+0.00029}$ & $0.02228_{-0.00015-0.00028}^{+0.00015+0.00030}$ & $0.02227_{-0.00015-0.00030}^{+0.00015+0.00030}$ & $0.02224_{-0.00014-0.00027}^{+0.00015+0.00028}$ \\
\hline $100 \theta_{M C}$ & $1.04101_{-0.00031-0.00060}^{+0.00032+0.00060}$ & $1.04079_{-0.00032-0.00063}^{+0.00033+0.00063}$ & $1.04077_{-0.00031-0.00061}^{+0.00031+0.00062}$ & $1.04076_{-0.00031-0.00061}^{+0.00032+0.00061}$ \\
\hline$\tau$ & $0.082_{-0.017-0.034}^{+0.017+0.034}$ & $0.078_{-0.018-0.034}^{+0.017+0.034}$ & $0.078_{-0.017-0.033}^{+0.017+0.033}$ & $0.079_{-0.017-0.034}^{+0.017+0.034}$ \\
\hline$n_{s}$ & $0.9698_{-0.004-0.008}^{+0.004+0.009}$ & $0.9667_{-0.0045-0.0089}^{+0.0045+0.0089}$ & $0.9658_{-0.0043-0.0083}^{+0.0043+0.0086}$ & $0.9660_{-0.0041-0.0081}^{+0.0041+0.0081}$ \\
\hline $\ln \left(10^{10} A_{s}\right)$ & $3.095_{-0.034-0.067}^{+0.034+0.066}$ & $3.090_{-0.034-0.067}^{+0.034+0.066}$ & $3.089_{-0.033-0.064}^{+0.033+0.064}$ & $3.090_{-0.033-0.067}^{+0.033+0.066}$ \\
\hline$w_{0}$ & $-1.056_{-0.196-0.344}^{+0.179+0.356}$ & $-0.607_{-0.186-0.336}^{+0.172+0.348}$ & $-0.919_{-0.085-0.138}^{+0.071+0.152}$ & $-0.902_{-0.078-0.126}^{+0.057+0.139}$ \\
\hline$w_{a}$ & $-1.500_{-0.571-1.275}^{+0.718+1.133}$ & $-0.955_{-0.388-0.848}^{+0.543+0.897}$ & $-0.399_{-0.172-0.391}^{+0.251+0.388}$ & $-0.252_{-0.089-0.309}^{+0.220+0.252}$ \\
\hline$\Omega_{m 0}$ & $0.207_{-0.009-0.016}^{+0.009+0.017}$ & $0.335_{-0.015-0.030}^{+0.015+0.031}$ & $0.300_{-0.007-0.013}^{+0.007+0.014}$ & $0.316_{-0.007-0.013}^{+0.006+0.014}$ \\
\hline$\sigma_{8}$ & $0.959_{-0.020-0.039}^{+0.020+0.040}$ & $0.812_{-0.017-0.033}^{+0.017+0.034}$ & $0.845_{-0.017-0.030}^{+0.015+0.032}$ & $0.827_{-0.016-0.030}^{+0.016+0.031}$ \\
\hline$H_{0}$ & $\begin{array}{c}82.57_{-1.65-3.10}^{+1.66+3.25} \\
\end{array}$ & $\begin{array}{c}65.19_{-1.48-2.65}^{+1.31+2.83} \\
\end{array}$ & $68.88_{-0.76-1.43}^{+0.73+1.47}$ & $67.11_{-0.69-1.37}^{+0.68+1.37}$ \\
\hline
\end{tabular}

summarized in the second column of Table III. In the top left panel of Fig. 6, we compare the constraints on the model parameters from the datasets from which one can clearly see that the inclusion of GW data significantly reduces the error bars on the model parameters. In particular, one can notice that both the datasets (CMB and $\mathrm{CMB}+\mathrm{GW}$ ) return very high values of the Hubble constant with similar mean values while the error bars on $H_{0}$ are reduced significantly after the inclusion of GW to CMB. As one can see, $H_{0}=82.78_{-8.34}^{+15.48}$ (68\% C.L., CMB) and $H_{0}=82.57_{-1.65}^{+1.66}(68 \%$ C.L., CMB + GW). It shows that the inclusion of GW reduces the error bars by a factor more than 5. In fact, for $68 \%$ upper C.L. errors, this reduction is very high. The dark energy equation of state at present, i.e., $w_{0}$ is constrained to be very close to the cosmological constant boundary $w_{0}=-1$ from both the datasets namely, $\mathrm{CMB}$ and $\mathrm{CMB}+\mathrm{GW}$. One can see that at $68 \%$ C.L. CMB data alone constrain $w_{0}=-1.058_{-0.550}^{+0.354}$ while for $\mathrm{CMB}+\mathrm{GW}, w_{0}=-1.056_{-0.196}^{+0.179}$ (68\% C.L.).
This clearly shows that the addition of GW to CMB significantly reduces the error bars on $w_{0}$, almost by a factor (not less than) of 2 and thus reflects the constraining power of GW. Concerning the other free parameter of the model, $w_{a}$, we find that CMB alone cannot constrain it while the inclusion of GW could constrain it well with $w_{a}=-1.500_{-0.571}^{+0.718}(68 \%$ C.L., CMB + GW). So, this clearly reflects the constraining power of GW.

We note that the constraints on $w_{a}$ are not so stringent due to high error bars. Furthermore, the correlations between the parameters (see the top left panel of Fig. 6) follow a similar trend as seen for the same datasets with the CPL model (i.e., top left panel of Fig. 3). Overall, the constraining power of $\mathrm{GW}$ is quite clear from the results.

When BAO is added to CMB (see the third column of Table III summarizing the results), we find that $H_{0}$ is significantly lowered with small error bars compared to the constraints from $\mathrm{CMB}$ giving $H_{0}=63.30_{-2.52}^{+1.87}$ (68\% C.L., CMB + BAO), and when the GW is added 

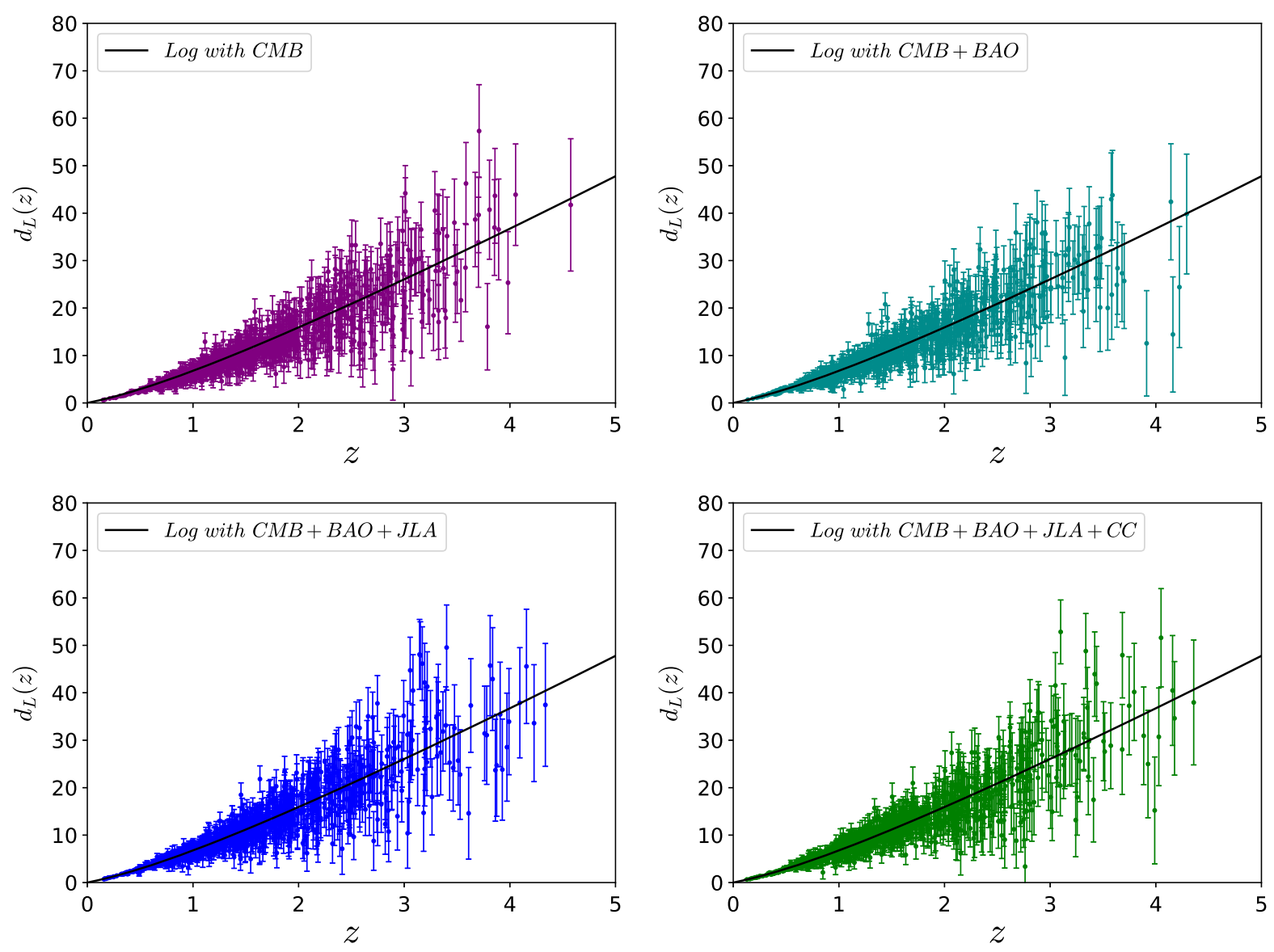

FIG. 5. For the fiducial logarithmic model, we first constrain the cosmological parameters using the datasets $\mathrm{CMB}$, $\mathrm{CMB}+\mathrm{BAO}$, $\mathrm{CMB}+\mathrm{BAO}+\mathrm{JLA}$, and $\mathrm{CMB}+\mathrm{BAO}+\mathrm{JLA}+\mathrm{CC}$ and then we use the best-fit values of the parameters for each dataset to generate the corresponding GW catalogue. Following this, in each panel we show $d_{L}(z)$ vs $z$ catalogue with the corresponding error bars for 1000 simulated GW events. The upper left and upper right panels respectively present the catalogue $\left(z, d_{L}(z)\right)$ with the corresponding error bars for 1000 simulated GW events derived using the CMB alone and CMB + BAO dataset. The lower left and lower right panels respectively present the catalogue $\left(z, d_{L}(z)\right)$ with the corresponding error bars for 1000 simulated GW events derived using the $\mathrm{CMB}+\mathrm{BAO}+\mathrm{JLA}$ and $\mathrm{CMB}+\mathrm{BAO}+\mathrm{JLA}+\mathrm{CC}$ datasets.

to $\mathrm{CMB}+\mathrm{BAO}$, the error bars are further decreased, but the mean value of $H_{0}$ slightly increases with $H_{0}=$ 65.19-1.48 (68\% C.L., CMB + BAO + GW). The constraints on $\Omega_{m 0}$ are significantly high for both the datasets $\left(\Omega_{m 0}=0.356_{-0.024}^{+0.026}\right.$ at $68 \%$ C.L. for CMB + BAO, and $\Omega_{m 0}=0.335_{-0.015}^{+0.015}$ at $68 \%$ C.L. for CMB + BAO $+\mathrm{GW}$ ), similar to what we found for the CPL parametrization with the same datasets (we refer to the third colum of Table II for comparisons). Concerning the key parameters $w_{0}, w_{a}$ of the model, we see that $w_{0}$ is very far from $w_{0}=-1$ and $w_{a}$ is very high (considering its magnitude). In Fig. 6 we have compared the constraints between the datasets from which we can see that the parameters are correlated with each other. This result has already been found for the CPL parametrization with the same datasets (compare the top right panels of Figs. 3 and 6).

We now discuss the next analyses with JLA. In particular, we focus on the constraints from $\mathrm{CMB}+\mathrm{BAO}+\mathrm{JLA}$ and $\mathrm{CMB}+\mathrm{BAO}+\mathrm{JLA}+\mathrm{GW}$. The results are summarized in the third column of Table III and in the bottom left panel of Fig. 6 we compare the constraints from the datasets. We see that the inclusion of JLA improves the constraints from $\mathrm{CMB}+\mathrm{BAO}$; that means the constraints from $\mathrm{CMB}+\mathrm{BAO}+\mathrm{JLA}$ are more stringent than $\mathrm{CMB}+$ $\mathrm{BAO}$ and the inclusion of GW gives more fine constraints on the parameters. Specifically, looking at the constraints on the Hubble constant given by $H_{0}=67.93_{-1.19}^{+1.11}$ $\left(68 \%\right.$ C.L., CMB + BAO + JLA) and $H_{0}=68.88_{-0.76}^{+0.73}$ (68\% C.L., CMB + BAO + JLA + GW), one can see that the inclusion of GW shifts $H_{0}$ towards its higher values with lower error bars. Concerning the dark energy equation of state at present, $w_{0}$, we see that for the $\mathrm{CMB}+\mathrm{BAO}+\mathrm{JLA}$ dataset, $w_{0}=-0.895_{-0.098}^{+0.084}(68 \%$ C.L. $)$ and $w_{0}=-0.919_{-0.085}^{+0.071}(68 \%$ C.L., CMB + BAO + JLA + $\mathrm{GW})$. It shows that the inclusion of GW shifts the dark energy equation of state towards the cosmological constant 

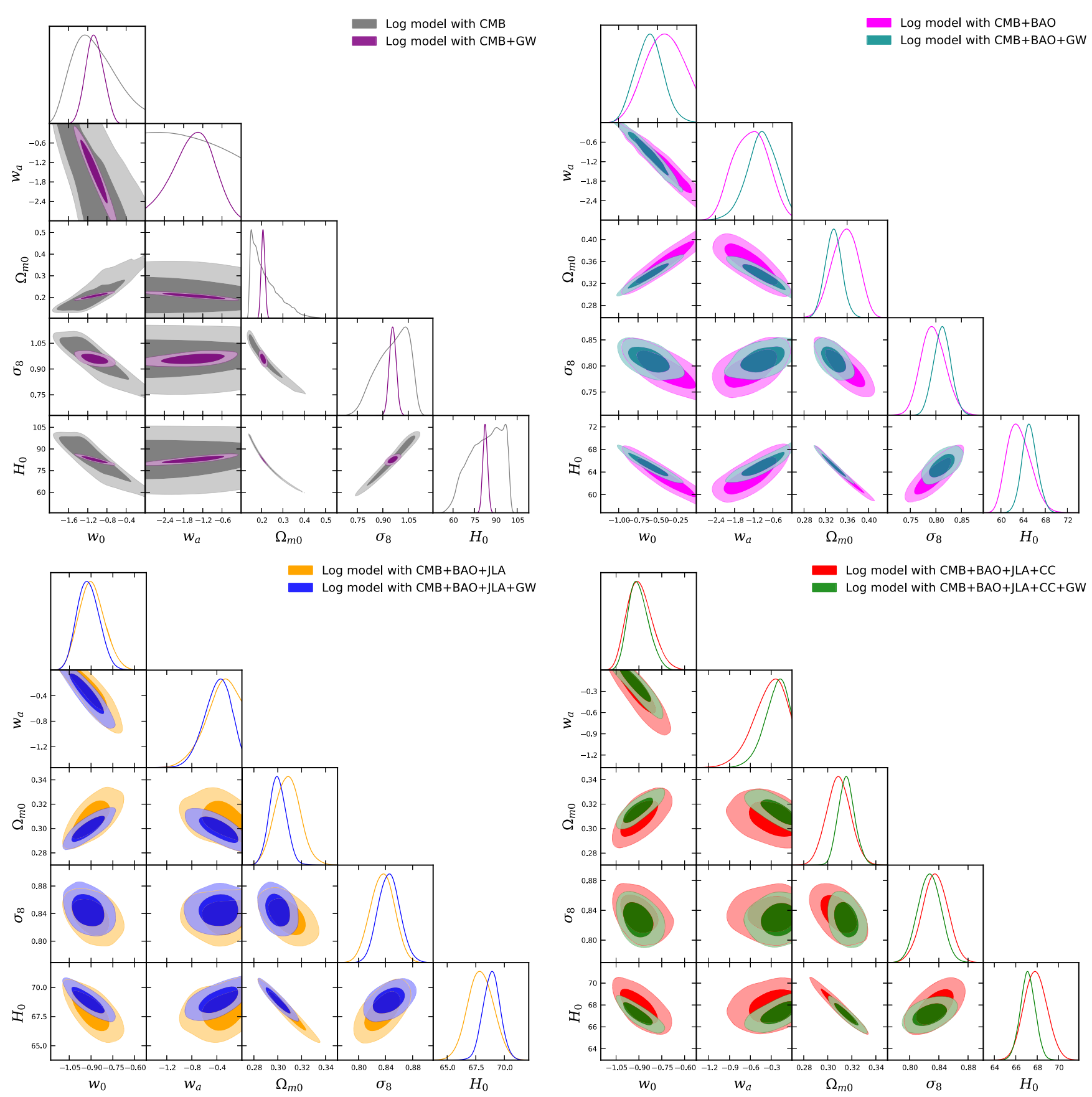

FIG. 6. In this figure we have shown the $68 \%$ and $95 \%$ confidence-level contour plots for various combinations of some selected parameters of the logarithmic parametrization (11) using different observational data in the presence (absence) of the GW data.

boundary with some improvements in the error bars. More precisely, looking at the $1 \mathrm{D}$ posterior distributions for $w_{0}$ shown in the bottom left panel of Fig. 6, one can see that the highest peaks of $w_{0}$ are quintessential. We also find that the constraints on $w_{a}$ are significantly lowered (considering its magnitude) compared to the previous two datasets, namely, CMB (and its companion $\mathrm{CMB}+\mathrm{GW}$ ) and $\mathrm{CMB}+\mathrm{BAO}$ (and its companion $\mathrm{CMB}+\mathrm{BAO}+\mathrm{GW})$. In particular, the estimations are $w_{a}=-0.365_{-0.083}^{+0.365}(68 \%$ C.L., CMB + BAO + JLA) and $w_{a}=-0.399_{-0.172}^{+0.251} \quad(68 \% \quad$ C.L., $\quad \mathrm{CMB}+\mathrm{BAO}+\mathrm{JLA}+$ GW). Now, finally, looking at the lower left panel of Fig. 6 one can say that the correlations between the parameters $\sigma_{8}$ and $w_{a}$ seem to be absent while the correlations (either positive or negative) with others still exist after the inclusion of JLA. We also remark that such correlations are not affected by the GW data.

We finish the observational analyses after the inclusion of the Hubble parameter measurements from $\mathrm{CC}$ to the previous dataset $\mathrm{CMB}+\mathrm{BAO}+\mathrm{JLA}$. The results are 

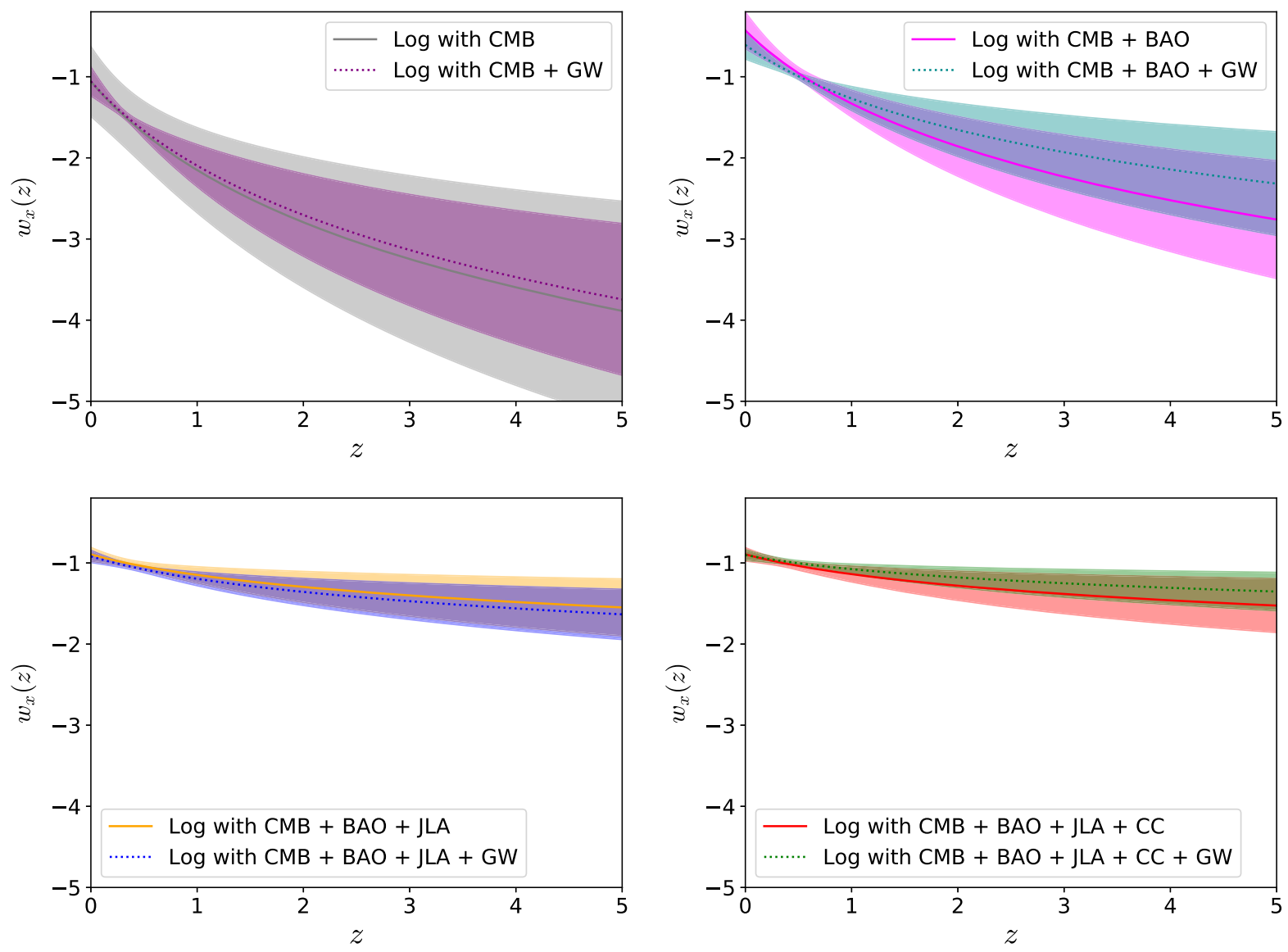

FIG. 7. The evolution of the dark energy equation of state for the logarithmic parametrization has been shown for different datasets taking the mean values of the key parameters $w_{0}$ and $w_{a}$ from the analyses with and without the GW data. The solid curves stand for the evolution of $w_{x}(z)$ for the standard cosmological probes while the dotted curves stand for the dataset in the presence of the GW data. The shaded regions show the $68 \%$ C.L. constraints on these two parameters.

summarized in the last column of Table III and the bottom right panel of Fig. 6 corresponds to the comparisons between the datasets. We find that almost all parameters are constrained in a similar way to $\mathrm{CMB}+\mathrm{BAO}+\mathrm{JLA}$ except the key parameters $w_{0}, w_{a}$ where we have some different observations. We find here for both the datasets $w_{0}>-1$ strictly at $68 \%$ C.L.; this is different from the previous analyses where for $\mathrm{CMB}+\mathrm{BAO}+\mathrm{JLA}+\mathrm{GW}$, $w_{0}<-1$ was allowed at $68 \%$ C.L. But of course the highest peaks of the 1D posterior distributions of $w_{0}$ for both $\mathrm{CMB}+\mathrm{BAO}+\mathrm{JLA}+\mathrm{CC}$ and its companion $\mathrm{CMB}+\mathrm{BAO}+\mathrm{JLA}+\mathrm{CC}+\mathrm{GW}$ are in favor of a quintessential dark energy at present. The parameter $w_{a}$ becomes more stringent than its estimation from $\mathrm{CMB}+$ $\mathrm{BAO}+\mathrm{JLA}$ reducing error bars: $w_{a}=-0.352_{-0.137}^{+0.293}$ $(68 \%$ C.L., $\mathrm{CMB}+\mathrm{BAO}+\mathrm{JLA}+\mathrm{CC})$ and $w_{a}=$ $-0.252_{-0.089}^{+0.220} \quad(68 \% \quad$ C.L., $\quad$ CMB + BAO + JLA + CC + $\mathrm{GW})$, which show that the standard cosmological probe (i.e., $\mathrm{CMB}+\mathrm{BAO}+\mathrm{JLA}+\mathrm{CC}$ ) allows $w_{a}=0$, in $68 \%$ C.L., but the inclusion of GW changes this conclusion favoring the dynamical dark energy for this parametrization within $68 \%$ C.L.
Last but not least, in Fig. 7, we have shown the evolution of the dark energy state for this parametrization using the mean values of $w_{0}$ and $w_{a}$ for the observational datasets employed in the work. The solid lines in each plot stand for the $w_{x}(z)$ curve using the usual cosmological probe and the dotted lines represent the evolution of $w_{x}(z)$ in the presence of the GW data. In each plot the shaded regions (with similar colors to the corresponding curves) present the $68 \%$ regions for the parameters $w_{0}, w_{a}$ corresponding to each dataset (with or without the GW data). This figure gives a qualitative nature of this dark energy equation of state in a nutshell. As one can see, the inclusion of GW to the standard cosmological data certainly improves the parameter space. The maximum effects of GW are visible with the CMB data alone.

\section{JBP parametrization}

We now discuss the observational constraints on the JBP parametrization (12) in order to investigate the effects of GW data on the cosmological parameters of this model. We first constrain this parametrization using the standard cosmological probes (summarized in the upper half of the 

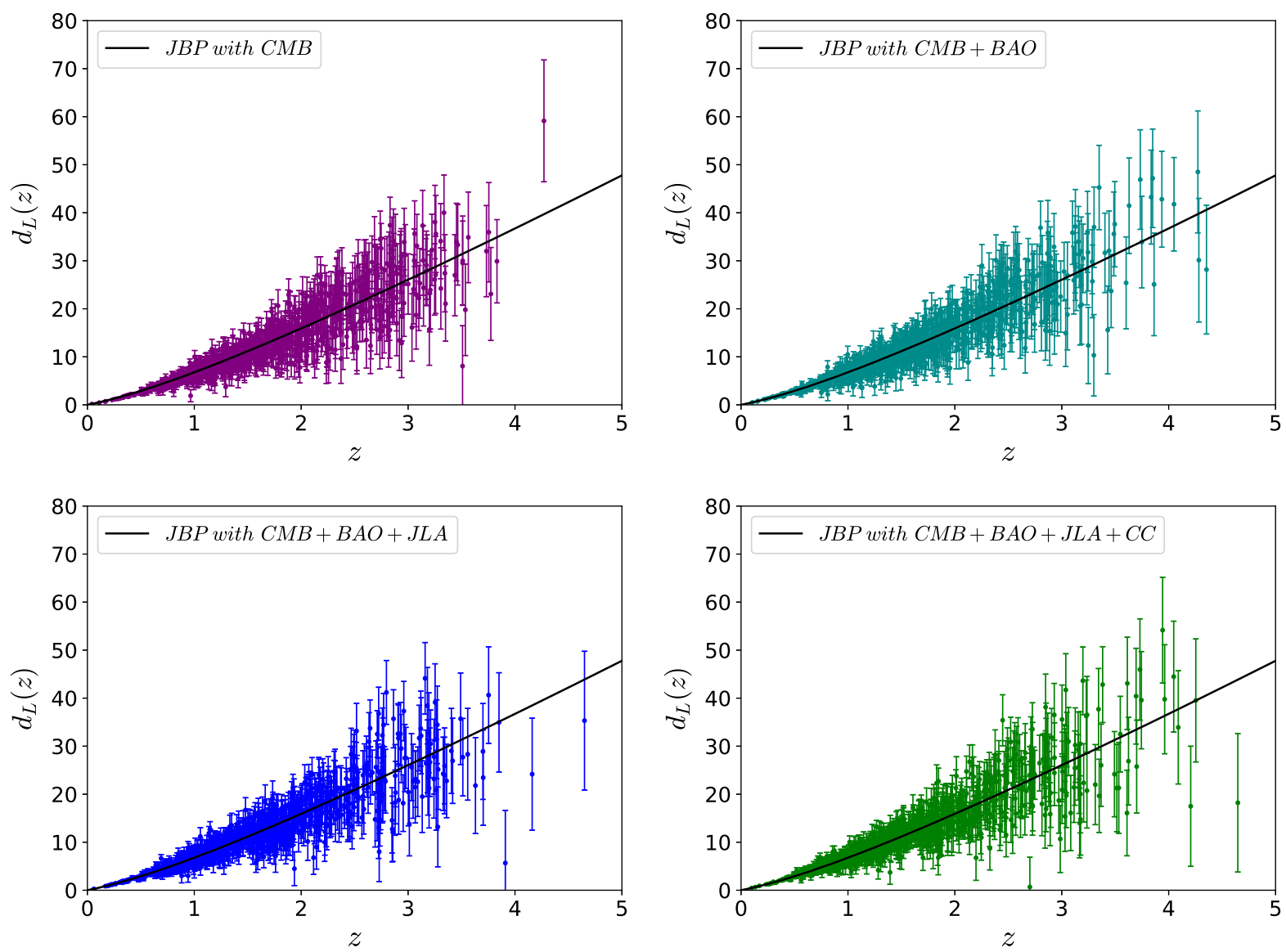

FIG. 8. For the fiducial JBP model, we first constrain the cosmological parameters using the datasets $\mathrm{CMB}$, CMB $+\mathrm{BAO}$, $\mathrm{CMB}+\mathrm{BAO}+\mathrm{JLA}$, and $\mathrm{CMB}+\mathrm{BAO}+\mathrm{JLA}+\mathrm{CC}$ and then we use the best-fit values of the parameters for each dataset to generate the corresponding GW catalogue. Following this, in each panel we show $d_{L}(z)$ vs $z$ catalogue with the corresponding error bars for 1000 simulated GW events. The upper left and upper right panels respectively present the catalogue $\left(z, d_{L}(z)\right)$ with the corresponding error bars for 1000 simulated GW events derived using the CMB alone and CMB + BAO dataset. The lower left and lower right panels respectively present the catalogue $\left(z, d_{L}(z)\right)$ with the corresponding error bars for 1000 simulated GW events derived using the $\mathrm{CMB}+\mathrm{BAO}+\mathrm{JLA}$ and $\mathrm{CMB}+\mathrm{BAO}+\mathrm{JLA}+\mathrm{CC}$ datasets.

Table IV), and then using the best-fit values of the model parameters, we generate the GW catalogue comprising 1000 simulated GW events. In Fig. 8, we have shown the relation $d_{L}(z)$ vs $z$ for the 1000 simulated GW events. Now, taking into account the simulated GW events with the standard cosmological probes, we have constrained the model parameters that are summarized in the lower half of Table II. Following a similar strategy, we display Fig. 9 that clearly depicts the effects of GW on the cosmological parameters.

We first discuss the constraints from the CMB data alone and the $\mathrm{CMB}+\mathrm{GW}$ dataset summarized in the second column of Table IV. In the top left panel of Fig. 9, we present the comparisons between the observational constraints obtained from these two datasets. Our analyses report that for both the datasets, $H_{0}$ assumes very high values with $H_{0}=84.01_{-7.82}^{+13.21}$ (68\% C.L., CMB) and $H_{0}=$ $82.73_{-0.54}^{+0.49}$ (68\% C.L., CMB + GW). Clearly, one can see that the inclusion of simulated GW data decreases the error bars on $H_{0}$ by a factor of at least 15 . This is one of the interesting conclusions and a similar conclusion has been found in earlier dark energy parametrizations such as CPL and logarithmic. The reduction in the error bars for other cosmological parameters is equally true after the inclusion of the simulated GW data; see the top left panel of Fig. 9 for a better viewing. Similar effects on the key two free parameters of this model, namely, $w_{0}$ and $w_{a}$, are observed. An interesting remark might be the allowance of phantom nature of $w_{0}$ by both the datasets, namely, CMB and $\mathrm{CMB}+\mathrm{GW}$ at more than $68 \%$ C.L. Moreover, from the highest peaks of the 1D posterior distributions of $w_{0}$ for both the datasets, namely, $\mathrm{CMB}$ and $\mathrm{CMB}+\mathrm{GW}$, the phantom nature of $w_{0}$ is strongly suggested. Concerning the $w_{a}$ parameter, we note that the addition of GW to the $\mathrm{CMB}$ in a similar fashion improves its parameter space (see the top left panel of Fig. 9).

The inclusion of BAO to the former datasets works in a similar fashion as observed in the previous two 

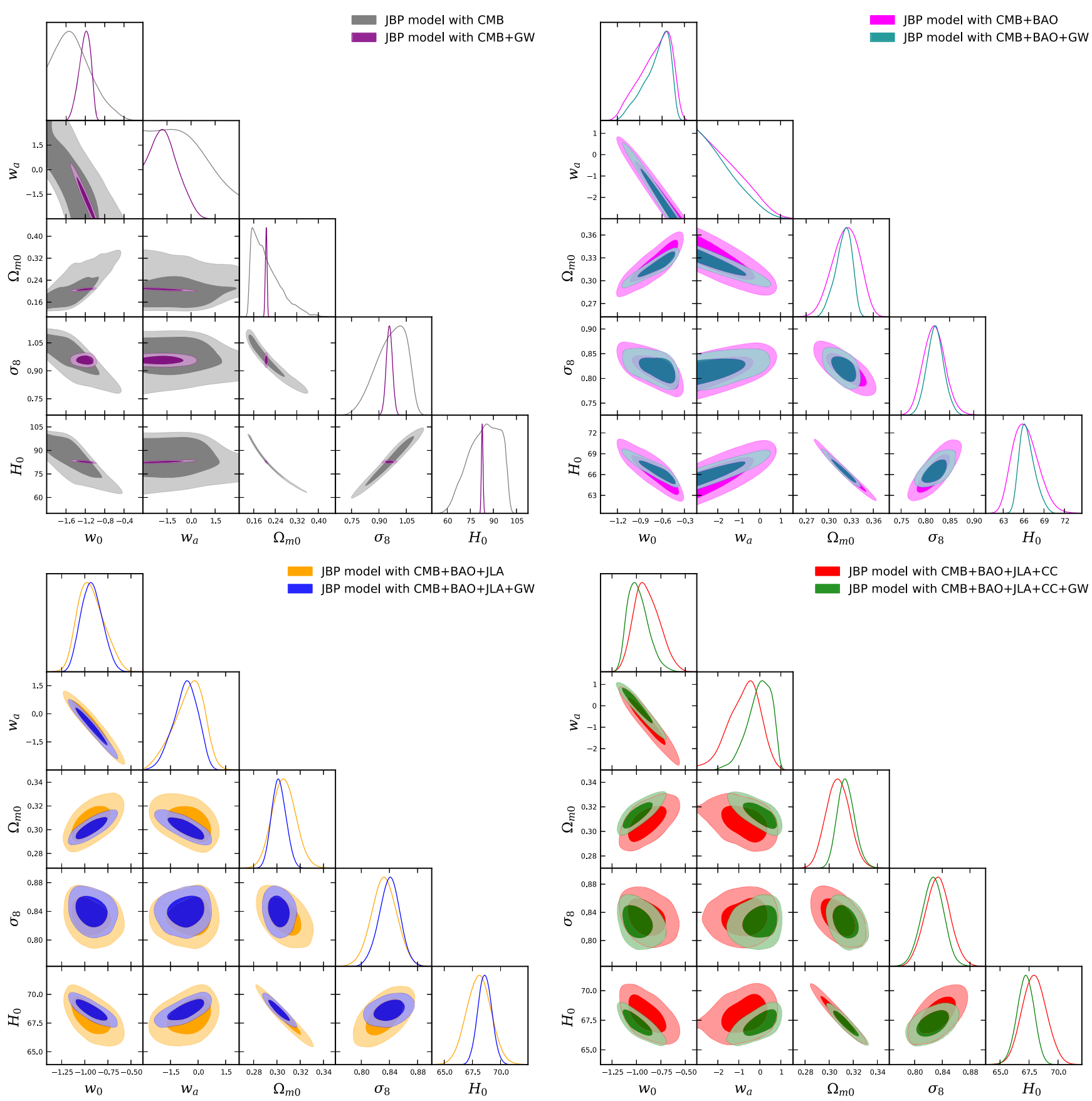

FIG. 9. Sixty-eight percent and 95\% C.L. contour plots for various combinations of some selected parameters of the JBP model (12) using different observational data in the presence (absence) of the GW data.

parametrizations. The results of the analyses are summarized in the third column of Table IV while we compare the constraints in the top right panel of Fig. 9. From this figure (i.e., the top right panel of Fig. 9), we can see that the parameters shown there are correlated with each other, where one may perhaps recognize that the correlation between $w_{a}$ and $\sigma_{8}$ is relatively low compared to other combinations in this figure. There is one more point that we should make here. Although $H_{0}$ assumes slightly lower values that the estimation from Planck's team, compared to the previous two dynamical dark energy parametrizations, $H_{0}$ is relatively higher, $H_{0}=66.29_{-2.26}^{+1.58}$ and $H_{0}=66.54_{-1.41}^{+0.89}$ (68\% C.L., CMB + BAO). Similar effects are seen in the estimations of the present-day matter density parameter $\Omega_{m 0}$.

Let us now discuss the observational constraints from $\mathrm{CMB}+\mathrm{BAO}+\mathrm{JLA}$ and its companion $\mathrm{CMB}+\mathrm{BAO}+$ JLA + GW. The fourth column of Table IV summarizes the constraints on the parameters and the bottom left panel of Fig. 9 corresponds to the comparison of the datasets. From the figure (i.e., bottom left panel of Fig. 9) we see that 
TABLE IV. Observational constraints at $68 \%$ and $95 \%$ confidence levels for the JBP parametrization (12) using various combinations of the observational data with and without the GW data. The upper panel represents the constraints on the model without the GW data while in the lower panel we present the corresponding constraints using the GW data. For the $w_{a}$ parameter the sign $<$ denotes that we report its $95 \%$ C.L. constraint. Here $\Omega_{m 0}$ is the present value of $\Omega_{m}=\Omega_{b}+\Omega_{c}$ and $H_{0}$ is in the units of km s $\mathrm{Mpc}^{-1}$.

\begin{tabular}{|c|c|c|c|c|}
\hline Parameters & CMB & $\mathrm{CMB}+\mathrm{BAO}$ & $\mathrm{CMB}+\mathrm{BAO}+\mathrm{JLA}$ & $\mathrm{CMB}+\mathrm{BAO}+\mathrm{JLA}+\mathrm{CC}$ \\
\hline$\overline{\Omega_{c} h^{2}}$ & $0.1191_{-0.0014-0.0028}^{+0.0014+0.0029}$ & $0.1187_{-0.0013-0.0024}^{+0.0013+0.0025}$ & $0.1188_{-0.0013-0.0026}^{+0.0013+0.0025}$ & $0.1189_{-0.0012-0.0025}^{+0.0013+0.0025}$ \\
\hline$\Omega_{b} h^{2}$ & $0.02228_{-0.00016-0.00031}^{+0.00015+0.0031}$ & $0.02229_{-0.00015-0.00029}^{+0.00015+0.00030}$ & $0.02229_{-0.00014-0.00028}^{+0.00013-0.0026}$ & $0.02229_{-0.00014-0.00030}^{+0.00014+0.00030}$ \\
\hline $100 \theta_{M C}$ & $1.04080_{-0.00033-0.00066}^{+0.00035+0.0064}$ & $1.04084_{-0.00032-0.00061}^{+0.00031+0.00063}$ & $1.04084_{-0.00030-0.00061}^{+0.00031+0.0063}$ & $1.04083_{-0.00030-0.00059}^{+0.00031+0.00063}$ \\
\hline$\tau$ & $0.076_{-0.018-0.035}^{+0.018+0.034}$ & $0.083_{-0.017-0.033}^{+0.017+0.033}$ & $0.081_{-0.017-0.035}^{+0.017+0.034}$ & $0.081_{-0.017-0.033}^{+0.018+0.033}$ \\
\hline$n_{s}$ & $0.9664_{-0.0046-0.0091}^{+0.0045+0.0090}$ & $0.9676_{-0.0044-0.0087}^{+0.0044+0.0085}$ & $0.9673_{-0.0043-0.0083}^{+0.0043+0.0084}$ & $0.9671_{-0.0044-0.0084}^{+0.0044+0.0089}$ \\
\hline $\ln \left(10^{10} A_{s}\right)$ & $3.084_{-0.035-0.068}^{+0.036+0.066}$ & $3.097_{-0.034-0.066}^{+0.034+0.064}$ & $3.093_{-0.034-0.068}^{+0.034+0.067}$ & $3.095_{-0.033-0.065}^{+0.034+0.066}$ \\
\hline$w_{0}$ & $-1.423_{-0.491-0.577}^{+0.220+0.674}$ & $-0.692_{-0.144-0.423}^{+0.279+0.346}$ & $-0.932_{-0.177-0.255}^{+0.115+0.293}$ & $-0.893_{-0.148-0.247}^{+0.120+0.268}$ \\
\hline$w_{a}$ & $<3$ & $<0.214$ & $-0.508_{-0.622-1.734}^{+1.017+1.424}$ & $-0.737_{-0.689-1.514}^{+0.839+1.446}$ \\
\hline$\Omega_{m 0}$ & $0.210_{-0.069-0.085}^{+0.027+0.115}$ & $0.323_{-0.017-0.036}^{+0.021+0.034}$ & $0.306_{-0.010-0.019}^{+0.010+0.021}$ & $0.307_{-0.010-0.018}^{+0.010+0.019}$ \\
\hline$\sigma_{8}$ & $0.967_{-0.062-0.161}^{+0.106+0.141}$ & $0.819_{-0.023-0.045}^{+0.022+0.045}$ & $0.832_{-0.018-0.035}^{+0.018+0.035}$ & $0.833_{-0.018-0.035}^{+0.018+0.035}$ \\
\hline$H_{0}$ & $84.01_{-7.82-18.75}^{+13.21+17.11}$ & $66.29_{-2.26-3.54}^{+1.58+3.80}$ & $68.07_{-1.09-2.15}^{+1.08+2.15}$ & $67.95_{-1.04-2.00}^{+1.05+2.09}$ \\
\hline Parameters & $\mathrm{CMB}+\mathrm{GW}$ & $\mathrm{CMB}+\mathrm{BAO}+\mathrm{GW}$ & $\mathrm{CMB}+\mathrm{BAO}+\mathrm{JLA}+\mathrm{GW}$ & $\mathrm{CMB}+\mathrm{BAO}+\mathrm{JLA}+\mathrm{CC}+\mathrm{GW}$ \\
\hline$\Omega_{c} h^{2}$ & $0.1182_{-0.0012-0.0023}^{+0.0011+0.0023}$ & $0.1186_{-0.0012-0.0025}^{+0.0012+0.0024}$ & $0.1190_{-0.0012-0.0024}^{+0.0012+0.0023}$ & $0.1189_{-0.0013-0.0024}^{+0.0013+0.0024}$ \\
\hline$\Omega_{b} h^{2}$ & $0.02238_{-0.00014-0.00027}^{+0.00013+0.00027}$ & $0.02232_{-0.00016-0.00028}^{+0.00014+0.00030}$ & $0.02229_{-0.00014-0.00029}^{+0.00014+0.00028}$ & $0.02225_{-0.00014-0.00029}^{+0.00014+0.00029}$ \\
\hline $100 \theta_{M C}$ & $1.04095_{-0.00031-0.00060}^{+0.00031+0.00059}$ & $1.04088_{-0.00031-0.00061}^{+0.00031+0.00060}$ & $1.04081_{-0.00031-0.00059}^{+0.00031+0.00059}$ & $1.04077_{-0.00032-0.00062}^{+0.00032+0.00061}$ \\
\hline$\tau$ & $0.080_{-0.017-0.033}^{+0.017+0.033}$ & $0.083_{-0.018-0.034}^{+0.018+0.034}$ & $0.081_{-0.016-0.034}^{+0.018+0.032}$ & $0.082_{-0.017-0.033}^{+0.017+0.032}$ \\
\hline$n_{s}$ & $0.9689_{-0.0042-0.0081}^{+0.0041+0.0081}$ & $0.9680_{-0.0042-0.0084}^{+0.0042+0.0081}$ & $0.9667_{-0.0042-0.0082}^{+0.0041+0.0084}$ & $0.9669_{-0.0042-0.0084}^{+0.0041+0.0087}$ \\
\hline $\ln \left(10^{10} A_{s}\right)$ & $3.092_{-0.033-0.065}^{+0.033+0.065}$ & $3.098_{-0.035-0.066}^{+0.035+0.065}$ & $3.094_{-0.032-0.066}^{+0.035+0.062}$ & $3.097_{-0.033-0.064}^{+0.032+0.064}$ \\
\hline$w_{0}$ & $-1.213_{-0.097-0.240}^{+0.152+0.218}$ & $-0.672_{-0.106-0.370}^{+0.234+0.286}$ & $-0.925_{-0.131-0.220}^{+0.108+0.225}$ & $-0.982_{-0.132-0.193}^{+0.080+0.215}$ \\
\hline$w_{a}$ & $<-0.126$ & $<-0.019$ & $-0.683_{-0.549-1.346}^{+0.828+1.234}$ & $-0.029_{-0.391-1.141}^{+0.755+1.003}$ \\
\hline$\Omega_{m 0}$ & $0.206_{-0.003-0.006}^{+0.003+0.006}$ & $0.320_{-0.009-0.023}^{+0.014+0.020}$ & $0.302_{-0.007-0.011}^{+0.006+0.012}$ & $0.314_{-0.008-0.014}^{+0.007+0.015}$ \\
\hline$\sigma_{8}$ & $0.957_{-0.016-0.033}^{+0.016+0.032}$ & $0.821_{-0.017-0.034}^{+0.017+0.036}$ & $0.840_{-0.014-0.030}^{+0.016+0.029}$ & $0.826_{-0.015-0.032}^{+0.017+0.030}$ \\
\hline$H_{0}$ & $82.73_{-0.54-0.97}^{+0.49+1.02}$ & $66.54_{-1.41-2.08}^{+0.89+2.42}$ & $68.60_{-0.60-1.19}^{+0.61+1.22}$ & $67.19_{-0.71-1.50}^{+0.79+1.40}$ \\
\hline
\end{tabular}

although the correlations between the parameters are present, $\sigma_{8}$ does not seem to be correlated with $w_{0}, w_{a}$; at least, a very mild correlation might be present that is not pronounced from the plots. The Hubble constant is shifted towards the higher values after the inclusion of GW and the error bars are reduced slightly. This feature has been observed in other parametrizations. Looking at the dark energy equation of state at present, $w_{0}$, we see that indeed the inclusion of GW improves the constraints on $w_{0}$ but not significantly. From the 1D posterior distributions of $w_{0}$ (see the bottom left panel of Fig. 9), it is indeed seen that the highest peaks of $w_{0}$ for both the datasets are very close to -1 ; that means the cosmological constant is favored. Furthermore, when GW data are added, a very minimal shift of the highest peak of $w_{0}$ towards the quintessence regime is observed. On the other hand, from the numerical estimations of the $w_{a}$ parameter, $w_{a}=-0.508_{-0.622}^{+1.017}$ $(68 \%$ C.L., CMB + BAO + JLA $)$ and $w_{a}=-0.683_{-0.549}^{+0.828}$ $(68 \%$ C.L., CMB + BAO + JLA + GW $)$, one can clearly see that the inclusion of JLA to the previous dataset $\mathrm{CMB}+\mathrm{BAO}$ reduces the magnitude of $w_{a}$. However, due to the very large error bars on $w_{a}$, the case $w_{a}=0$ is definitely allowed within $68 \%$ C.L. We also notice that for $\mathrm{CMB}+\mathrm{BAO}+\mathrm{JLA}+\mathrm{GW}$, the highest peak of the $1 \mathrm{D}$ posterior distribution of $w_{a}$ (see the bottom left panel of Fig. 9) is shifted towards more negative values compared to the highest peak of $w_{a}$ for CMB $+\mathrm{BAO}+\mathrm{JLA}$. This is true because here $w_{0}$ and $w_{a}$ are negatively correlated; see again the $\left(w_{0}, w_{a}\right)$ plane shown in the bottom left panel of Fig. 9.

Finally, we come up with the last two analyses, namely, $\mathrm{CMB}+\mathrm{BAO}+\mathrm{JLA}+\mathrm{CC}$ and $\mathrm{CMB}+\mathrm{BAO}+\mathrm{JLA}+\mathrm{CC}+$ $\mathrm{GW}$. The results are summarized in the last column of Table IV and in the bottom right corner of Fig. 9 we compare these datasets. The only surprising result is observed in the constraints on one of the key parameters of this model, namely, $w_{a}$ where we find that the $68 \%$ constraints on this parameter are $w_{a}=-0.737_{-0.689}^{+0.839}$ 
$(\mathrm{CMB}+\mathrm{BAO}+\mathrm{JLA}+\mathrm{CC})$ and $w_{a}=-0.029_{-0.391}^{+0.755}$. One can clearly visualize the effect of GW on this parameter where precisely the estimated value of $w_{a}$ is remarkably lowered together with significant reduction of its error bars. This is an interesting result and we remark that the previous two dynamical dark energy parametrizations, namely, CPL and logarithmic, did not exhibit such behavior. We further note that although the mean values of $w_{0}$ attained from both the datasets are nonphantom, from the 1D posterior distributions of $w_{0}$ (bottom right corner of Fig. 9), we see that the inclusion of GW to $\mathrm{CMB}+\mathrm{BAO}+\mathrm{JLA}+\mathrm{CC}$ shifts the highest peak of $w_{0}$ towards the phantom regime.

We close this analysis with Fig. 10, where we present the qualitative evolution of the dark energy equation of state for this parametrization using the mean values of $w_{0}$ and $w_{a}$ obtained from the observational datasets employed in the work. The solid lines in each plot stand for the $w_{x}(z)$ curve using the usual cosmological probe and the dotted lines represent the evolution of $w_{x}(z)$ in the presence of the GW data. In each plot the shaded regions (with similar colors to the corresponding curves) present the $68 \%$ regions for the parameters $w_{0}, w_{a}$ corresponding to each dataset (with or
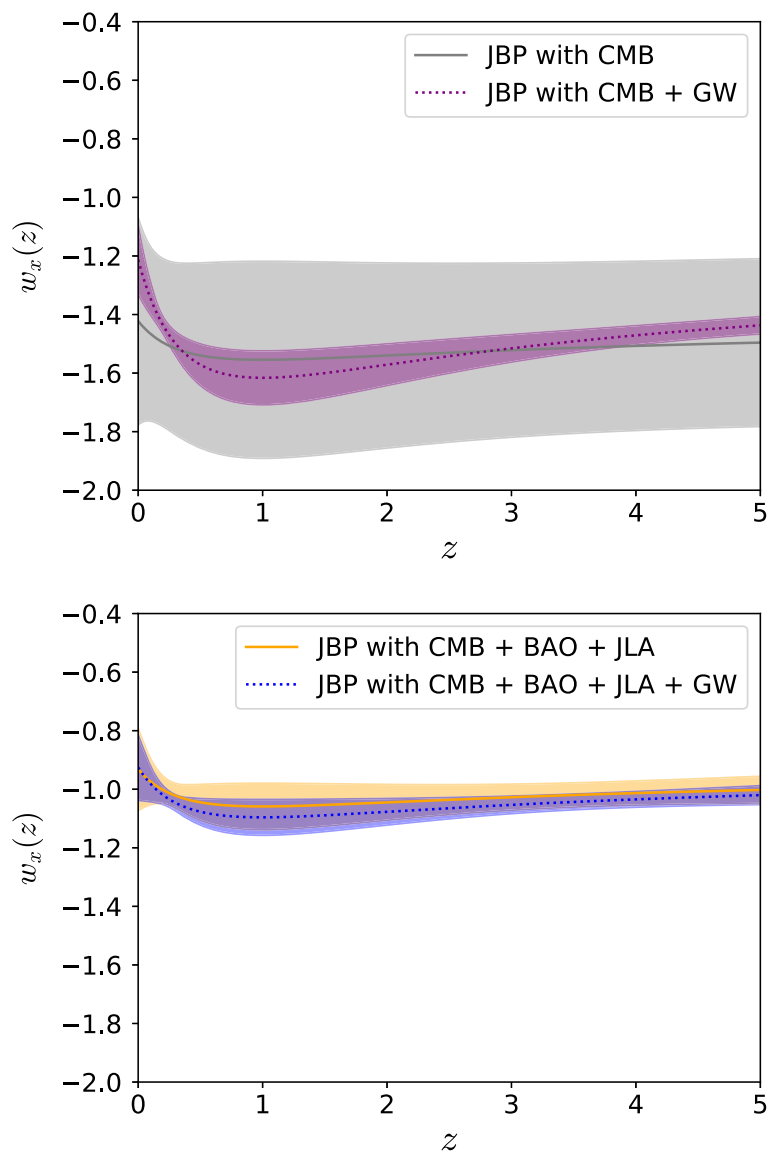

without the GW data). From the graphs, one can clearly see how GW data affect the cosmological parameters. The maximum effects of $\mathrm{GW}$ are seen from the $\mathrm{CMB}$ alone case. We note that the dotted curve for $\mathrm{CMB}+\mathrm{BAO}+$ $\mathrm{JLA}+\mathrm{CC}+\mathrm{GW}$ (see the bottom right graph of Fig. 10) is almost a straight line, $w_{x}(z)=-1$.

\section{Barboza-Alcaniz parametrization}

Finally, we confront the Barboza-Alcaniz parametrization (13) following a similar pattern performed for another three dynamical dark energy parametrizations.

We use the standard cosmological probes, such as $\mathrm{CMB}$, $\mathrm{BAO}$, JLA, and $\mathrm{CC}$, to constrain the parameter space of this model (summarized in the upper half of Table V), and then using the best-fit values of the model parameters, we have generated the GW catalogue comprising 1000 simulated GW events. In Fig. 11, we have shown the relation $d_{L}(z)$ vs $z$ for the 1000 simulated GW events. Now, using the simulated GW events with the standard cosmological probes, we have constrained this parametrization. The summary of the observational constraints on this model
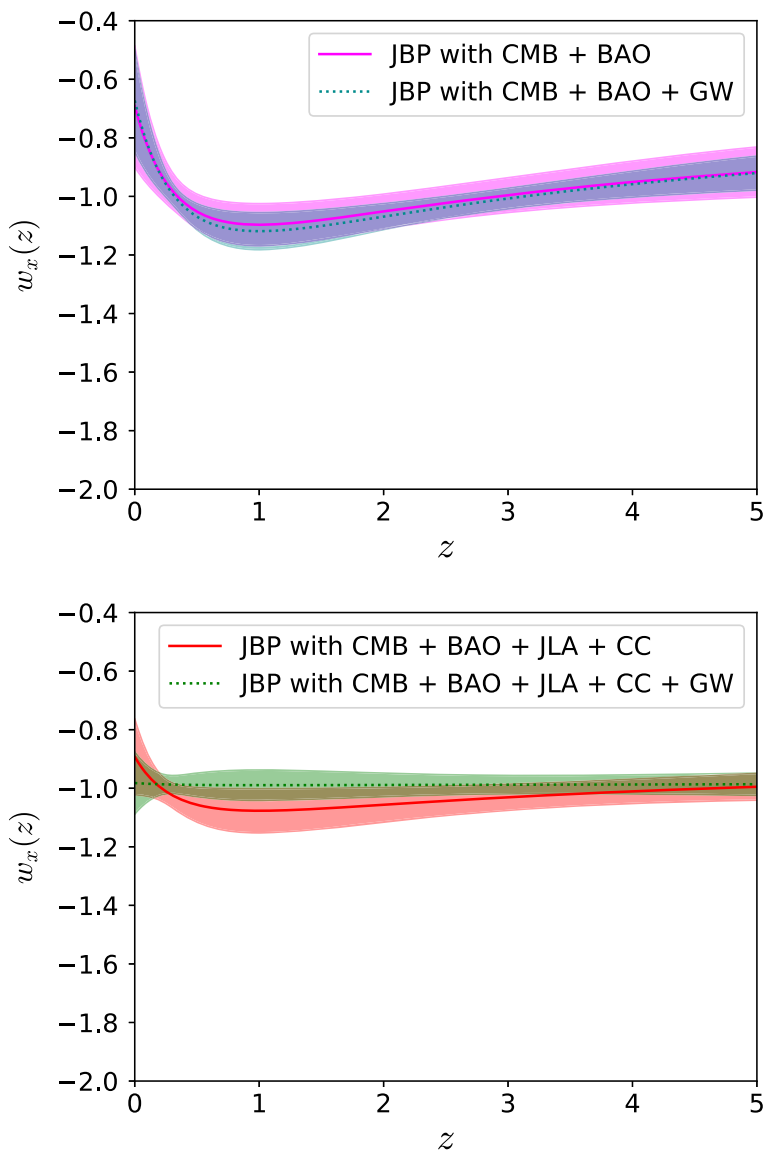

FIG. 10. The evolution of the dark energy equation of state for the JBP parametrziation has been shown for different datasets taking the mean values of the key parameters $w_{0}$ and $w_{a}$ from the analyses with and without the $\mathrm{GW}$ data. The solid curves stand for the evolution of $w_{x}(z)$ for the standard cosmological probes while the dotted curves stand for the dataset in the presence of the GW data. The shaded regions show the $68 \%$ C.L. constraints on these two parameters. 
TABLE V. Observational constraints at $68 \%$ and $95 \%$ confidence levels for the Barboza-Alcaniz parametrization (13) using various combinations of the observational data with and without the GW data. The upper panel represents the constraints on the model without the GW data while in the lower panel we present the corresponding constraints using the GW data. For the CMB-only case the upper limits of the $w_{a}$ parameter at $68 \%$ and $95 \%$ C.L. are reported. Here, $\Omega_{m 0}$ is the present value of $\Omega_{m}=\Omega_{b}+\Omega_{c}$ and $H_{0}$ is in units of $\mathrm{km} \mathrm{s}^{-1} \mathrm{Mpc}^{-1}$.

\begin{tabular}{|c|c|c|c|c|}
\hline Parameters & $\mathrm{CMB}$ & $\mathrm{CMB}+\mathrm{BAO}$ & $\mathrm{CMB}+\mathrm{BAO}+\mathrm{JLA}$ & $\mathrm{CMB}+\mathrm{BAO}+\mathrm{JLA}+\mathrm{CC}$ \\
\hline$\overline{\Omega_{c} h^{2}}$ & $0.1191_{-0.0014-0.0027}^{+0.0014+0.0028}$ & $0.1190_{-0.0014-0.0025}^{+0.0013+0.0026}$ & $0.1192_{-0.0013-0.0027}^{+0.0013+0.0027}$ & $0.1189_{-0.0013-0.0025}^{+0.0012+0.0024}$ \\
\hline$\Omega_{b} h^{2}$ & $0.02228_{-0.00015-0.00030}^{+0.00016+0.00031}$ & $0.02227_{-0.00015-0.00028}^{+0.00015+0.00029}$ & $0.02226_{-0.00015-0.00029}^{+0.00015+00030}$ & $0.02229_{-0.00015-0.00028}^{+0.00015+0.00030}$ \\
\hline $100 \theta_{M C}$ & $1.04079_{-0.00033-0.00065}^{+0.00033+0.00063}$ & $1.04080_{-0.00031-0.00061}^{+0.00032+0.00061}$ & $1.04077_{-0.00032-0.00064}^{+0.00032+0.00065}$ & $1.04081_{-0.00031-0.00061}^{+0.00031+0.00061}$ \\
\hline$\tau$ & $0.076_{-0.017-0.033}^{+0.017+0.034}$ & $0.079_{-0.017-0.034}^{+0.017+0.033}$ & $0.079_{-0.018-0.034}^{+0.018+0.033}$ & $0.082_{-0.019-0.033}^{+0.017+0.034}$ \\
\hline$n_{s}$ & $0.9665_{-0.0045-0.0089}^{+0.0046+0.0087}$ & $0.9668_{-0.0045-0.0084}^{+0.0042+0.0090}$ & $0.9662_{-0.0044-0.0087}^{+0.0044+0.0090}$ & $0.9673_{-0.0041-0.0085}^{+0.0042+0.0087}$ \\
\hline $\ln \left(10^{10} A_{s}\right)$ & $3.085_{-0.033-0.065}^{+0.033+0.066}$ & $3.091_{-0.033-0.066}^{+0.033+0.064}$ & $3.091_{-0.034-0.067}^{+0.034+0.065}$ & $3.097_{-0.034-0.064}^{+0.033+0.067}$ \\
\hline$w_{0}$ & $-1.386_{-0.556-0.614}^{+0.203+0.761}$ & $-0.692_{-0.374-0.486}^{+0.215+0.589}$ & $-0.898_{-0.090-0.174}^{+0.093+0.182}$ & $-0.933_{-0.066-0.139}^{+0.064+0.142}$ \\
\hline$w_{a}$ & $<-0.038<0.613$ & $-0.509_{-0.282-0.920}^{+0.577+0.722}$ & $-0.263_{-0.165-0.388}^{+0.211+0.361}$ & $-0.173_{-0.109-0.281}^{+0.137+0.235}$ \\
\hline$\Omega_{m 0}$ & $0.215_{-0.075-0.092}^{+0.024+0.136}$ & $0.334_{-0.038-0.055}^{+0.024+0.061}$ & $0.308_{-0.010-0.018}^{+0.009+0.020}$ & $0.307_{-0.010-0.019}^{+0.009+0.019}$ \\
\hline$\sigma_{8}$ & $0.964_{-0.058-0.178}^{+0.114+0.146}$ & $0.811_{-0.027-0.055}^{+0.027+0.053}$ & $0.835_{-0.018-0.036}^{+0.018+0.037}$ & $0.835_{-0.017-0.034}^{+0.017+0.034}$ \\
\hline$H_{0}$ & $83.55_{-7.20-20.82}^{+14.43+17.75}$ & $65.41_{-3.03-5.33}^{+3.00+5.39}$ & $67.91_{-1.06-2.10}^{+1.05+2.07}$ & $67.98_{-0.99-2.05}^{+1.00+2.03}$ \\
\hline Parameters & $\mathrm{CMB}+\mathrm{GW}$ & $\mathrm{CMB}+\mathrm{BAO}+\mathrm{GW}$ & $\mathrm{CMB}+\mathrm{BAO}+\mathrm{JLA}+\mathrm{GW}$ & $\mathrm{CMB}+\mathrm{BAO}+\mathrm{JLA}+\mathrm{CC}+\mathrm{GW}$ \\
\hline$\Omega_{c} h^{2}$ & $0.1185_{-0.0012-0.0024}^{+0.0013+0.0024}$ & $0.1193_{-0.0013-0.0027}^{+0.0013+0.0026}$ & $0.1191_{-0.0012-0.0025}^{+0.0013+0.0026}$ & $0.1190_{-0.0013-0.0024}^{+0.0013+0.0025}$ \\
\hline$\Omega_{b} h^{2}$ & $0.02234_{-0.00014-0.00027}^{+0.00014+0.00027}$ & $0.02226_{-0.00015-0.00029}^{+0.00015+0.00029}$ & $0.02226_{-0.00015-0.00029}^{+0.00014+0.00030}$ & $0.02224_{-0.00014-0.00028}^{+0.00015+0.0028}$ \\
\hline $100 \theta_{M C}$ & $1.04090_{-0.00031-0.00061}^{+0.00031+0.00060}$ & $1.04077_{-0.00033-0.00063}^{+0.00032+0.00064}$ & $1.04078_{-0.00031-0.00062}^{+0.00031+0.0060}$ & $1.04076_{-0.00031-0.00060}^{+0.00031+0.0062}$ \\
\hline$\tau$ & $0.079_{-0.017-0.032}^{+0.016+0.032}$ & $0.078_{-0.017-0.035}^{+0.018+0.033}$ & $0.080_{-0.016-0.032}^{+0.016+0.032}$ & $0.081_{-0.017-0.034}^{+0.017+0.033}$ \\
\hline$n_{s}$ & $0.9679_{-0.0042-0.0079}^{+0.0042+0.0081}$ & $0.9660_{-0.0044-0.0088}^{+0.0044+0.0086}$ & $0.9665_{-0.0043-0.0084}^{+0.0043+0.0084}$ & $0.9666_{-0.0044-0.0082}^{+0.0043+0.0084}$ \\
\hline $\ln \left(10^{10} A_{s}\right)$ & $3.089_{-0.032-0.063}^{+0.032+0.062}$ & $3.089_{-0.033-0.068}^{+0.034+0.065}$ & $3.091_{-0.032-0.064}^{+0.032+0.063}$ & $3.094_{-0.034-0.066}^{+0.033+0.065}$ \\
\hline$w_{0}$ & $-1.253_{-0.153-0.255}^{+0.131+0.281}$ & $-0.711_{-0.155-0.256}^{+0.128+0.284}$ & $-0.925_{-0.070-0.136}^{+0.070+0.142}$ & $-1.000_{-0.075-0.144}^{+0.074+0.150}$ \\
\hline$w_{a}$ & $-0.516_{-0.295-0.669}^{+0.372+0.637}$ & $-0.523_{-0.213-0.517}^{+0.296+0.479}$ & $-0.186_{-0.130-0.287}^{+0.151+0.258}$ & $0.004_{-0.123-0.274}^{+0.142+0.247}$ \\
\hline$\Omega_{m 0}$ & $0.210_{-0.008-0.014}^{+0.007+0.015}$ & $0.328_{-0.013-0.025}^{+0.012+0.026}$ & $0.309_{-0.006-0.013}^{+0.006+0.013}$ & $0.312_{-0.007-0.014}^{+0.007+0.014}$ \\
\hline$\sigma_{8}$ & $0.955_{-0.019-0.036}^{+0.019+0.037}$ & $0.818_{-0.017-0.033}^{+0.017+0.034}$ & $0.833_{-0.015-0.029}^{+0.015+0.030}$ & $0.828_{-0.015-0.029}^{+0.015+0.031}$ \\
\hline$H_{0}$ & $82.12_{-1.32-2.69}^{+1.34+2.54}$ & $65.88_{-1.17-2.31}^{+1.20+2.35}$ & $67.83_{-0.65-1.27}^{+0.64+1.30}$ & $67.46_{-0.70-1.36}^{+0.69+1.42}$ \\
\hline
\end{tabular}

after the inclusion of the simulated GW data is shown in the lower half of Table V.

In Fig. 12 we present the graphical behavior between the free parameters of the model aiming to display the effects of GW on the cosmological parameters. From Table V, we again see that the inclusion of simulated GW data remarkably decreases the error bars on the parameters. Apart from that, this parametrization gives some interesting features that are described soon.

Following the similar pattern, we begin the analyses using the $\mathrm{CMB}$ data and $\mathrm{CMB}+\mathrm{GW}$ data. The results can be found in the second column of Table $\mathrm{V}$ and for these datasets, we have made a comparison in the top left panel of Fig. 12. From the analyses, one can visualize that the estimations of the Hubble constant from both the datasets are quite high, similar to what we have found in previous three dynamical dark energy parametrizations. For this parametrization, we find that $H_{0}=83.55_{-7.20}^{+14.43}$ (68\% C.L., $\mathrm{CMB}$ ) and $H_{0}=82.12_{-1.32}^{+1.34}$ (68\% C.L., $\left.\mathrm{CMB}+\mathrm{GW}\right)$.
As one can see, inclusion of $\mathrm{GW}$ to the $\mathrm{CMB}$ reduces the error bars almost by a factor of 6 (for $68 \%$ lower error bar on $H_{0}$ ) and almost by a factor of 10 (for $68 \%$ upper error bar on $H_{0}$ ). The reduction of error bars is also true for other model parameters. We note also that in a similar fashion, the constraints on the two key parameters of this model, namely, $w_{0}$ and $w_{a}$, are equally improved after the inclusion of GW data. However, for both the datasets, that means $\mathrm{CMB}$ and $\mathrm{CMB}+\mathrm{GW}, w_{0}$ remains in the phantom regime (i.e., $w_{0}<-1$ ) at more than $68 \%$ C.L. In fact, the highest peaks of the $1 \mathrm{D}$ posterior distributions for both the datasets are in the phantom regime. For a better visualization on the improvements of the parameters, we refer to the top left panel of Fig. 12. From this figure (top left panel of Fig. 12), one can clearly see how the inclusion of $\mathrm{GW}$ to the $\mathrm{CMB}$ improves the parameter space. We also comment that the estimation of $\Omega_{m 0}$ is small for both the datasets and this is also found for the other three models as well. 

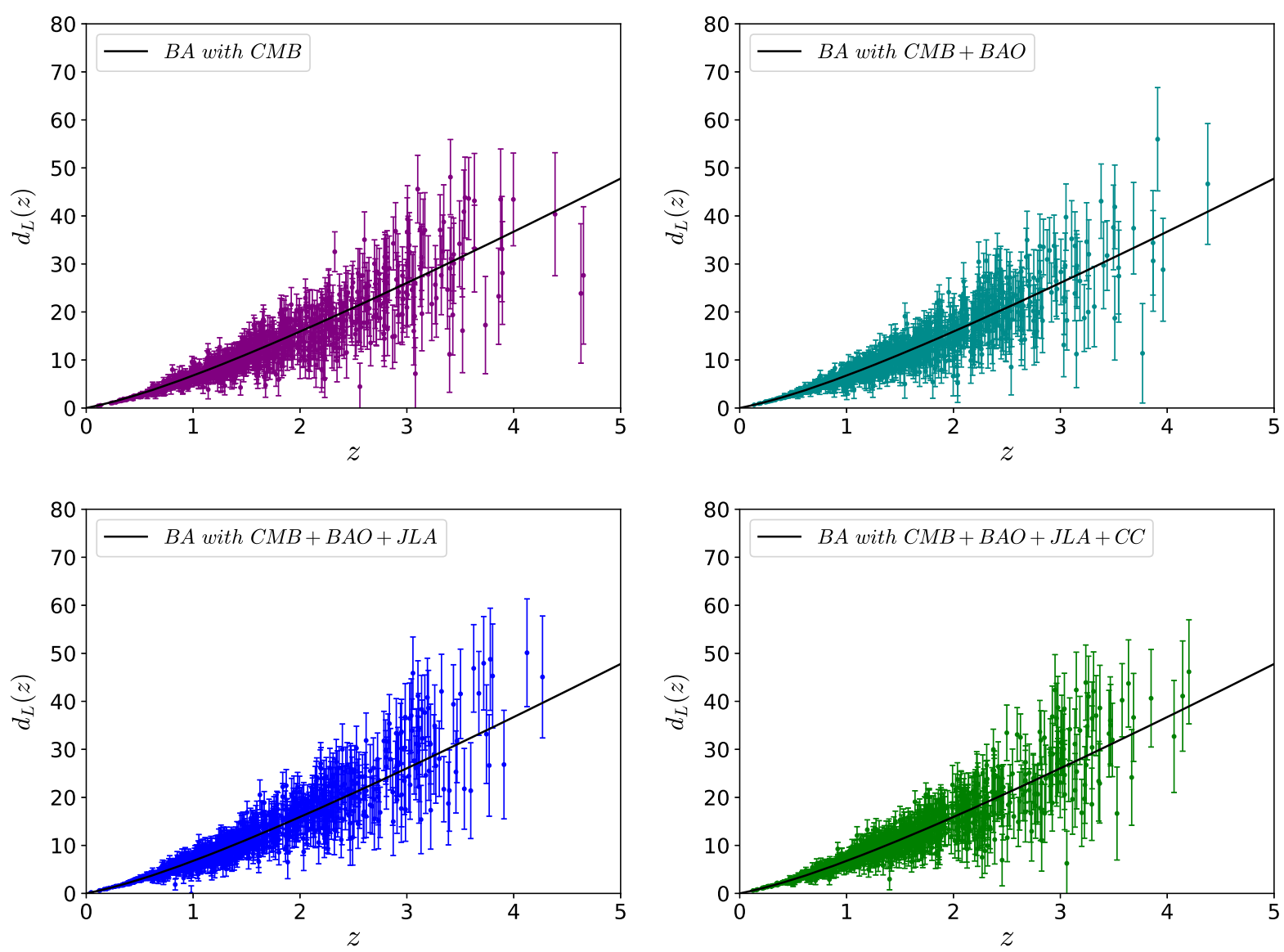

FIG. 11. For the fiducial BA model, we first constrain the cosmological parameters using the datasets $\mathrm{CMB}$, CMB $+\mathrm{BAO}$, $\mathrm{CMB}+\mathrm{BAO}+\mathrm{JLA}$, and $\mathrm{CMB}+\mathrm{BAO}+\mathrm{JLA}+\mathrm{CC}$ and then we use the best-fit values of the parameters for each dataset to generate the corresponding GW catalogue. Following this, in each panel we show $d_{L}(z)$ vs $z$ catalogue with the corresponding error bars for 1000 simulated GW events. The upper left and upper right panels respectively present the catalogue $\left(z, d_{L}(z)\right)$ with the corresponding error bars for 1000 simulated GW events derived using the CMB alone and CMB + BAO dataset. The lower left and lower right panels respectively present the catalogue $\left(z, d_{L}(z)\right)$ with the corresponding error bars for 1000 simulated GW events derived using the $\mathrm{CMB}+\mathrm{BAO}+\mathrm{JLA}$ and $\mathrm{CMB}+\mathrm{BAO}+\mathrm{JLA}+\mathrm{CC}$ datasets.

For the next analyses with $\mathrm{BAO}$, that means focusing on the combined analyses $\mathrm{CMB}+\mathrm{BAO}$ and $\mathrm{CMB}+\mathrm{BAO}+$ $\mathrm{GW}$; we do not find anything that is worth reporting. The results can be found from the third column of Table V and the comparison between the constraints on the model parameters using various datasets is shown in the top right plot of Fig. 12.

After the inclusion of JLA, results summarized in the fourth column of Table $\mathrm{V}$, we find that quintessence dark energy (i.e., $w_{0}>-1$ ) is preferred by the dataset $\mathrm{CMB}+$ $\mathrm{BAO}+\mathrm{JLA}$, and this remains so at $68 \%$ C.L. In addition, the inclusion of $\mathrm{GW}$ to this dataset does not alter this conclusion, meaning that within 68\% C.L., $w_{0}>-1$. We note that the highest peaks of the 1D posterior distributions of $w_{0}$ are bent towards the quintessence regime. Concerning the $w_{a}$ parameter, we find that the constraints are small (in magnitude) compared to the previsous datasets and also compared to the previous three models. We see that $w_{a}=-0.263_{-0.165}^{+0.211}$ (68\% C.L.,
$\mathrm{CMB}+\mathrm{BAO}+\mathrm{JLA})$ and $w_{a}=-0.186_{-0.130}^{+0.151}(68 \%$ C.L., $\mathrm{CMB}+\mathrm{BAO}+\mathrm{JLA}+\mathrm{GW})$. Thus, we see that both the datasets prefer $w_{a} \neq 0$, at least in $68 \%$ C.L. We refer to the bottom left panel of Fig. 12 for a comparison of the model parameters constraints obtained from different datasets.

Finally, we consider the last two combinations, namely, $\mathrm{CMB}+\mathrm{BAO}+\mathrm{JLA}+\mathrm{CC}$ and $\mathrm{CMB}+\mathrm{BAO}+\mathrm{JLA}+$ $\mathrm{CC}+\mathrm{GW}$. In the last column of Table $\mathrm{V}$, we have summarized the results and in the bottom right panel of Fig. 12 we have compared the cosmological constraints for this parametrization obtained from both the datasets. These analyses give some interesting results. Concerning the present value of the dark energy equation of state, $w_{0}$, we see that for the dataset $\mathrm{CMB}+\mathrm{BAO}+\mathrm{JLA}+\mathrm{CC}$, $w_{0}>-1$ and it remains so at more than $68 \%$ C.L., while after the inclusion of GW, this result is completely changed with the possibility of $w_{0}<-1$ at more than $68 \%$ C.L. More interestingly, for the dataset $\mathrm{CMB}+\mathrm{BAO}+\mathrm{JLA}+$ $\mathrm{CC}+\mathrm{GW}, w_{0}=-1.000_{-0.075}^{+0.074}$ at $68 \%$ C.L. In addition to 

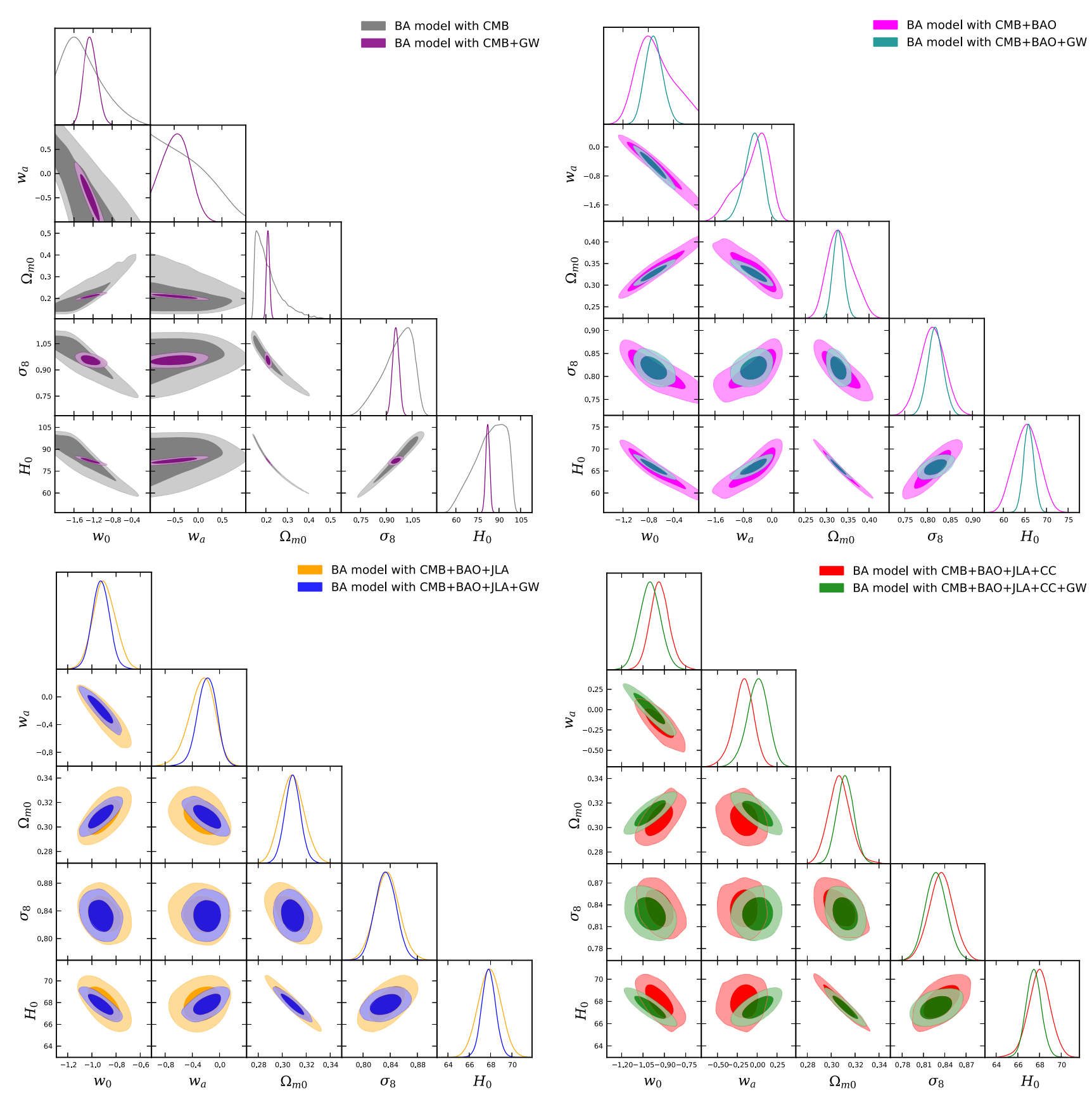

FIG. 12. Sixty-eight percent and 95\% C.L. contour plots for various combinations of some selected parameters of the BA model (13) using different observational data in the presence (absence) of the GW data.

that, after the inclusion of $\mathrm{GW}$, that means for the dataset $\mathrm{CMB}+\mathrm{BAO}+\mathrm{JLA}+\mathrm{CC}+\mathrm{GW}, w_{a}=0.004_{-0.123}^{+0.142}$ (68\% C.L.). Thus, from the overall constraints on both $w_{0}$ and $w_{a}$ for this model, one can clearly say that forecasting with future gravitational waves data strongly hints towards the $\Lambda \mathrm{CDM}$ type cosmology; however, looking at the constraints on $w_{a}$ for $\mathrm{CMB}+\mathrm{BAO}+\mathrm{JLA}+\mathrm{CC}+\mathrm{GW}$, the presence of large error bars implies that the observational data allow $w_{a} \neq 0$ as well.

Finally, using the mean values of $\left(w_{0}, w_{a}\right)$ from all the datasets, in Fig. 13 we depict the evolution of the dark energy equation of state $w_{x}(z)$ for this model. The solid lines in each plot stand for the $w_{x}(z)$ curve for the usual cosmological probe and the dotted lines depict the evolution of $w_{x}(z)$ in the presence of the GW data. In each plot the shaded regions (with similar colors to the corresponding curves) present the $68 \%$ regions for the parameters $w_{0}, w_{a}$ corresponding to each dataset (with or without the GW data). A quite interesting scenario we observe from the right plot of the lower panel of Fig. 13 (see the dotted curve in this plot) is that the mean curve for $w_{x}(z)$ is exactly equal to the cosmological constant, $w_{x}(z)=-1$. 

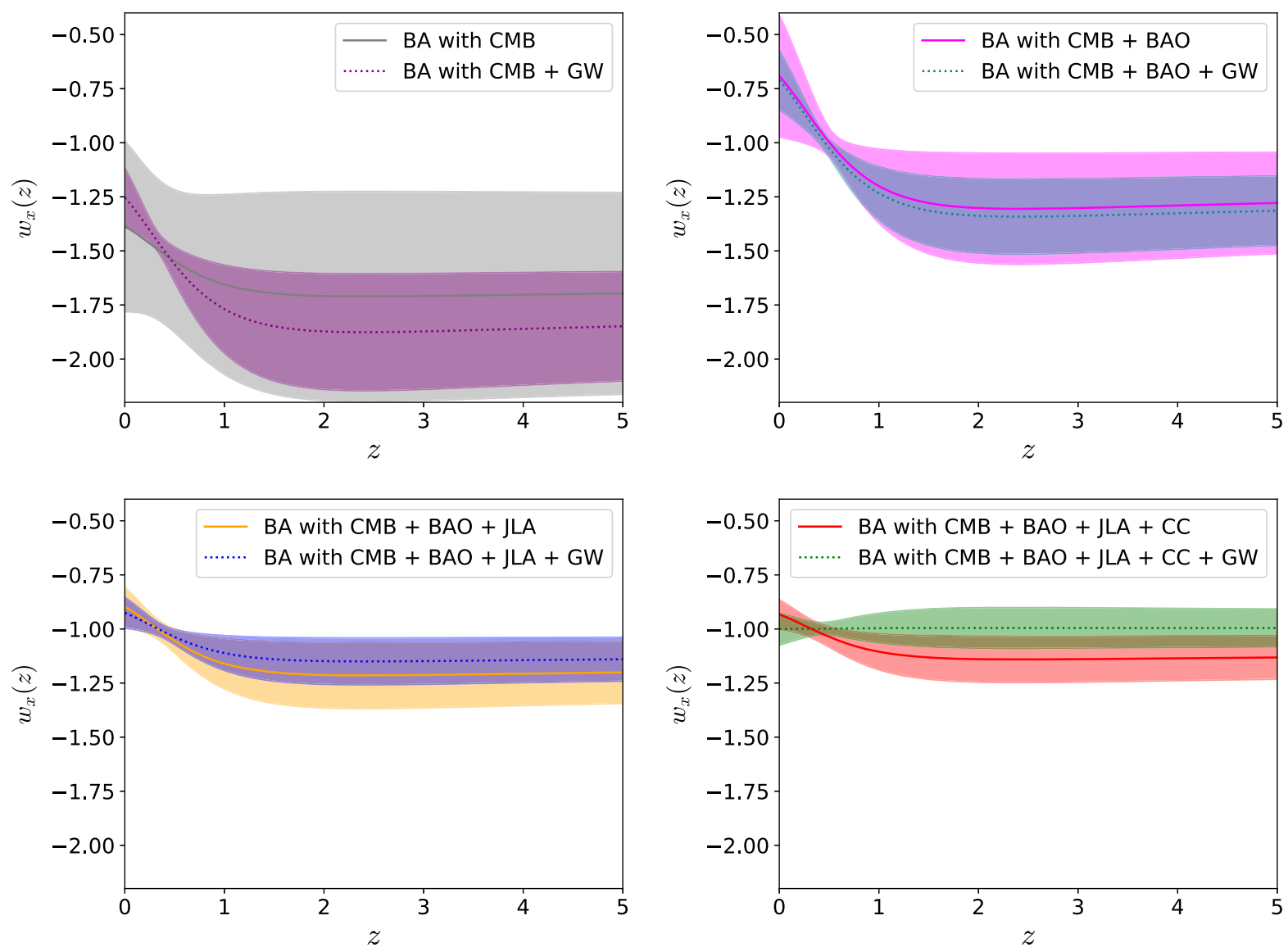

FIG. 13. The evolution of the dark energy equation of state for the BA parametrization has been shown for different datasets taking the mean values of the key parameters $w_{0}$ and $w_{a}$ from the analyses with and without the GW data. The solid curves stand for the evolution of $w_{x}(z)$ for the standard cosmological probes while the dotted curves stand for the dataset in the presence of the GW data. The shaded regions show the $68 \%$ C.L. constraints on these two parameters.

\section{CONCLUDING REMARKS}

The detection of gravitational waves has thrilled the scientific community by offering a new window of tests that may shine some light on the nature of gravity, dark matter, and dark energy. In the present work we investigate how GW data could bring further cosmological constraints to a class of dynamical dark energy models. In particular, we use 1000 simulated GW data from the Einstein Telescope (we refer the readers to Sec. III for detailed discussions on how the $\mathrm{GW}$ catalogue can be generated for any fiducial cosmological model).

In order to proceed we first consider four dynamical dark energy models characterized by their equation of state, namely, Chevallier-Polarski-Linder parametrization [Eq. (10)], logarithmic paramerization [Eq. (11)], Jassal-BaglaPadmanabhan parametrization [Eq. (12)], and BarbozaAlcaniz parametrization [Eq. (13)], and constrain them using the standard cosmological probes such as CMB, $\mathrm{BAO}$, JLA, and CC. Then considering the corresponding parametrizations as the fiducial models and using the best-fit values of the model parameters from each dataset (for a quick look at the best-fit values of the model parameters, see
Tables VII, VIII, IX, and X given in the Appendix), we generate the corresponding $\mathrm{GW}$ catalogue for the next step. In this way, we generate 1000 simulated GW events from the present dynamical models. Now, along with the 1000 simulated GW data from the Einstein Telescope, we include the aforementioned standard cosmological probes, namely, CMB, BAO, JLA, and CC, in order to understand the constraining power of GW data in the context of dynamical dark energy models.

The results of these four dynamical dark energy parametrizations are shown in Table II (CPL), Table III (logarithmic), Table IV (JBP), and Table V (BA). Further, the comparisons between the results of the observational constraints obtained from different cosmological datasets (with and without the GW data) have been graphically shown in Fig. 3 (CPL), Fig. 6 (logarithmic), Fig. 9 (JBP), and Fig. 12 (BA). For a quick review on some key parameters of these dark energy parametrizations, namely, $w_{0}, w_{a}$, and $H_{0}$, one can see Table VI.

Concerning the CPL parametrization [Eq. (10)], we find that the effects of GW are clearly pronounced, and such effects are clearly recognized if one looks at the constraints from $\mathrm{CMB}$ and $\mathrm{CMB}+\mathrm{GW}$ datasets. In particular, one can 
TABLE VI. Reporting of the $68 \%$ C.L. constraints on some key parameters of all the dark energy parametrizations, namely, $w_{0}, w_{a}$, and $H_{0}$ before and after the inclusion of $\mathrm{GW}$ to the standard cosmological probes. Let us note that here $\mathrm{C}=\mathrm{CMB}, \mathrm{CG}=\mathrm{CMB}+\mathrm{GW}$, $\mathrm{CB}=\mathrm{CMB}+\mathrm{BAO}, \quad \mathrm{CBG}=\mathrm{CMB}+\mathrm{BAO}+\mathrm{GW}, \quad \mathrm{CBJ}=\mathrm{CMB}+\mathrm{BAO}+\mathrm{JLA}, \quad \mathrm{CBJG}=\mathrm{CMB}+\mathrm{BAO}+\mathrm{JLA}+\mathrm{GW}$, $\mathrm{CBJC}=\mathrm{CMB}+\mathrm{BAO}+\mathrm{JLA}+\mathrm{CC}$, and $\mathrm{CBJCW}=\mathrm{CMB}+\mathrm{BAO}+\mathrm{JLA}+\mathrm{CC}+\mathrm{GW}$.

\begin{tabular}{|c|c|c|c|}
\hline Parameters & [C] [CG] & {$[\mathrm{CBJ}][\mathrm{CBJG}]$} & [CBJC] [CBJCG] \\
\hline$w_{0}(\mathrm{CPL})$ & {$\left[-1.218_{-0.597}^{+0.302}\right]\left[-1.168_{-0.212}^{+0.180}\right]$} & {$\left[-0.524_{-0.236}^{+0.374}\right]\left[-0.465_{-0.200}^{+0.189}\right]\left[-0.909_{-0.123}^{+0.095}\right]\left[-0.904_{-0.080}^{+0.070}\right]$} & {$\left[-0.909_{-0.116}^{+0.099}\right]\left[-0.902_{-0.062}^{+0.064}\right]$} \\
\hline$w_{a}(\mathrm{CPL})$ & {$[<-0.446]\left[-1.081_{-0.640}^{+0.842}\right]$} & {$\left[-1.403_{-1.021}^{+0.731}\right]\left[-1.523_{-0.562}^{+0.642}\right]\left[-0.409_{-0.277}^{+0.517}\right]\left[-0.256_{-0.227}^{+0.263}\right]$} & {$\left[-0.399_{-0.297}^{+0.423}\right]\left[-0.373_{-0.226}^{+0.263}\right]$} \\
\hline$H_{0}(\mathrm{CPL})$ & {$\left[83.06_{-7.98}^{+15.10}\right]\left[80.75_{-1.92}^{+1.71}\right]$} & {$\left[64.36_{-3.23}^{+2.05}\right]\left[63.77_{-1.52}^{+1.37}\right] \quad\left[67.94_{-1.08}^{+1.09}\right]\left[66.98_{-0.55}^{+0.55}\right]$} & {$\left[67.92_{-1.09}^{+1.09}\right]\left[67.72_{-0.35}^{+0.36}\right]$} \\
\hline$w_{0}(\log )$ & {$\left[-1.058_{-0.550}^{+0.354}\right]\left[-1.056_{-0.196}^{+0.179}\right]$} & {$\left[-0.429_{-0.223}^{+0.265}\right]\left[-0.607_{-0.186}^{+0.172}\right]\left[-0.895_{-0.098}^{+0.084}\right]\left[-0.919_{-0.085}^{+0.071}\right]$} & {$\left[-0.894_{-0.097}^{+0.072}\right]\left[-0.902_{-0.078}^{+0.057}\right]$} \\
\hline$w_{a}(\log )$ & [Unconstrained $]\left[-1.500_{-0.571}^{+0.718}\right]$ & {$\left[-1.301_{-0.570}^{+0.549}\right]\left[-0.955_{-0.388}^{+0.543}\right]\left[-0.365_{-0.083}^{+0.365}\right]\left[-0.399_{-0.172}^{+0.251}\right]$} & {$\left[-0.352_{-0.137}^{+0.293}\right]\left[-0.252_{-0.089}^{+0.220}\right]$} \\
\hline$H_{0}(\log )$ & {$\left[82.78_{-8.34}^{+15.48}\right]\left[82.57_{-1.65}^{+1.66}\right]$} & {$\left[63.30_{-2.52}^{+1.87}\right]\left[65.19_{-1.48}^{+1.31}\right]$} & {$\left[67.84_{-1.14}^{+1.05}\right]\left[67.11_{-0.69}^{+0.68}\right]$} \\
\hline$w_{0}(\mathrm{JBP})$ & {$\left[-1.423_{-0.491}^{+0.220}\right]\left[-1.213_{-0.097}^{+0.152}\right]$} & {$\left[-0.692_{-0.144}^{+0.279}\right]\left[-0.672_{-0.106}^{+0.234}\right]\left[-0.932_{-0.177}^{+0.115}\right]\left[-0.925_{-0.131}^{+0.108}\right]$} & {$\left[-0.893_{-0.148}^{+0.120}\right]\left[-0.982_{-0.132}^{+0.080}\right]$} \\
\hline$w_{a}(\mathrm{JBP})$ & {$[<0.19]\left[-1.614_{-1.100}^{+0.593}\right]$} & {$\left[-1.618_{-1.382}^{+0.417}\right]\left[-1.786_{-1.214}^{+0.321}\right]\left[-0.508_{-0.622}^{+1.017}\right]\left[-0.683_{-0.549}^{+0.828}\right]$} & {$\left[-0.737_{-0.689}^{+0.839}\right]\left[-0.029_{-0.391}^{+0.755}\right]$} \\
\hline$H_{0}(\mathrm{JBP})$ & {$\left[84.01_{-7.82}^{+13.21}\right]\left[82.73_{-0.54}^{+0.49}\right]$} & {$\left[66.29_{-2.26}^{+1.58}\right]\left[66.54_{-1.41}^{+0.89}\right] \quad\left[68.07_{-1.09}^{+1.08}\right]\left[68.60_{-0.60}^{+0.61}\right]$} & {$\left[67.95_{-1.04}^{+1.05}\right]\left[67.19_{-0.71}^{+0.79}\right]$} \\
\hline$w_{0}(\mathrm{BA})$ & {$\left[-1.386_{-0.556}^{+0.203}\right]\left[-1.253_{-0.153}^{+0.131}\right]$} & {$\left[-0.692_{-0.374}^{+0.215}\right]\left[-0.711_{-0.155}^{+0.128}\right]\left[-0.898_{-0.090}^{+0.093}\right]\left[-0.925_{-0.070}^{+0.070}\right]$} & {$\left[-0.933_{-0.066}^{+0.064}\right]\left[-1.000_{-0.075}^{+0.074}\right]$} \\
\hline$w_{a}(\mathrm{BA})$ & {$[<-0.038]\left[-0.516_{-0.295}^{+0.372}\right]$} & {$\left[-0.509_{-0.282}^{+0.577}\right]\left[-0.523_{-0.213}^{+0.296}\right]\left[-0.263_{-0.165}^{+0.211}\right]\left[-0.186_{-0.130}^{+0.151}\right]$} & {$\left[-0.173_{-0.109}^{+0.137}\right]\left[0.004_{-0.123}^{+0.142}\right]$} \\
\hline$H_{0}(\mathrm{BA})$ & {$\left[83.55_{-7.20}^{+14.43}\right]\left[82.12_{-1.32}^{+1.34}\right]$} & {$\left[65.41_{-3.03}^{+3.00}\right]\left[65.88_{-1.17}^{+1.20}\right] \quad\left[67.91_{-1.06}^{+1.05}\right]\left[67.83_{-0.65}^{+0.64}\right]$} & {$\left[67.98_{-0.99}^{+1.00}\right]\left[67.46_{-0.70}^{+0.69}\right]$} \\
\hline
\end{tabular}

see that $H_{0}=83.06_{-7.98}^{+15.10}(68 \%$ C.L., CMB $)$ and $H_{0}=$ $80.75_{-1.92}^{+1.71}(68 \%$ C.L., CMB $+\mathrm{GW})$. This clearly shows that the addition of $\mathrm{GW}$ to $\mathrm{CMB}$ reduces the error bars on $H_{0}$ in a significant way. Regarding other parameters, we also find that the dataset $\mathrm{CMB}+\mathrm{GW}$ improves them compared to their constraints from $\mathrm{CMB}$ alone. The improvements are significantly visible from some parameters; see the 1D posterior distributions as well as the 2D contour plots shown in the upper left panel of Fig. 3. However, for other datasets, such as $\mathrm{CMB}+\mathrm{BAO}+\mathrm{GW}, \mathrm{CMB}+\mathrm{BAO}+\mathrm{JLA}+$ $\mathrm{GW}$, and $\mathrm{CMB}+\mathrm{BAO}+\mathrm{JLA}+\mathrm{CC}+\mathrm{GW}$, the effects of $\mathrm{GW}$ is of course seen but not much compared to what we observed for the CMB $+\mathrm{GW}$ dataset. Now, concerning the present value of the dark energy equation of state, $w_{0}$, we see that the inclusion of GW significantly improves its parameter space by reducing its error bars. For the CMB alone case we see that the highest peaks of the 1D marginalized posterior distributions of $w_{0}$ (upper left panel of Fig. 3) are bent towards the phantom regime while for the remaining three cases, the highest peaks of the $1 \mathrm{D}$ marginalized posterior distributions of $w_{0}$ are bent towards the quintessence regime. While statistically, within $68 \%$ C.L., all four combinations allow $w_{0}>-1$ phase. Regarding the remaining free parameter of this model, namely, $w_{a}$, its improvements after the inclusion of GW are similarly visible; see for instance the 1D posterior distributions for $w_{a}$ for all the datasets (see Fig. 3). We remark that the highest peaks of the posterior distributions for $w_{a}$ are never 0 , which goes in favor of the dynamical dark energy equation of state.

Now, for the logarithmic parametrization [Eq. (11)] we have almost similar behavior to the CPL model [(10)].
The improvements of the parameters after the inclusion of GW are clearly visible; see the 1D posterior distributions of various parameters and the $2 \mathrm{D}$ contour plots shown in Fig. 6. One important remark for this parametrization is that for $\mathrm{CMB}$ data, $w_{a}$ is unconstrained while the addition of $\mathrm{GW}$ to $\mathrm{CMB}$ becomes able to constrain it.

For JBP parametrization [Eq. (12)] the reduction of error bars in the presence of GW follows a similar pattern as observed in CPL and logarithmic parametrizations. We refer to Fig. 9 (JBP parametrization) for a better understanding on how GW improves the cosmological constraints. Similar effects on the two key parameters of this model, namely, $w_{0}$ and $w_{a}$, are observed and we again refer to $1 \mathrm{D}$ posterior distributions of the parameters in Fig. 9. For the $w_{0}$ parameter, its highest peaks in the 1D posterior distributions are bent towards the quintessence regime. For the $w_{a}$ parameter we note that the final combination with $\mathrm{GW}$, which means the dataset $\mathrm{CMB}+\mathrm{BAO}+\mathrm{JLA}+$ $\mathrm{CC}+\mathrm{GW}$, returns its extremely lower mean value with $w_{a}=-0.029_{-0.391}^{+0.755}(68 \%$ C.L.) compared to its constraint obtained from the usual dataset $\mathrm{CMB}+\mathrm{BAO}+\mathrm{JLA}+$ CC: $w_{a}=-0.737_{-0.689}^{+0.839}$ (68\% C.L.).

For the last parametrization, i.e., BA parametrization [Eq. (13)], the improvements of the entire parameter space due to the inclusion of $\mathrm{GW}$ are evident from Table $\mathrm{X}$ and from Fig. 12. We also refer to Table VI in order to see how the key parameters $w_{0}$ and $w_{a}$ are affected due to the inclusion of GW. We see that the behavior of $w_{a}$ is different, which distinguishes it from other dark energy parametrizations. We notice that due to the addition of $\mathrm{GW}$ to the usual cosmological probes, the mean values of $w_{a}$ obtained from 
the standard cosmological probes become half with improvements in the parameter space $(68 \%$ constraints: $w_{a}=-0.263_{-0.165}^{+0.211}$ for $\mathrm{CMB}+\mathrm{BAO}+\mathrm{JLA}$, while $w_{a}=$ $-0.186_{-0.130}^{+0.151}$ for $\left.\mathrm{CMB}+\mathrm{BAO}+\mathrm{JLA}+\mathrm{GW}\right)$ or even smaller than half $\left(68 \%\right.$ constraints: $w_{a}=-0.173_{-0.109}^{+0.137}$ for $\mathrm{CMB}+\mathrm{BAO}+\mathrm{JLA}+\mathrm{CC}$, whereas $w_{a}=0.004_{-0.123}^{+0.142}$ for $\mathrm{CMB}+\mathrm{BAO}+\mathrm{JLA}+\mathrm{CC}+\mathrm{GW})$. Finally, we remark that for the last observational combination with $\mathrm{GW}$, that means for $\mathrm{CMB}+\mathrm{BAO}+\mathrm{JLA}+\mathrm{CC}+\mathrm{GW}$, the $68 \%$ C.L. constraints on $w_{0}$ and $w_{a}$ are $w_{0}=-1.000_{-0.075}^{+0.074}$ and $w_{a}=0.004_{-0.123}^{+0.142}$. This reflects its closeness to the $\Lambda$ type cosmology; however, due to the large error bars on $w_{a}$, its dynamical character is certainly allowed within $68 \%$ C.L.

Thus, our results clearly indicate that the future GW data may significantly affect the cosmological parameters, providing stringent constraints on them by reducing their error bars in a remarkable way. Although one may argue that for the above dark energy parametrizations, the future constraints from GW seem to prefer a quintessential dark energy (for BA parametrization, the last combination $\mathrm{CMB}+\mathrm{BAO}+\mathrm{JLA}+\mathrm{CC}+\mathrm{GW}$ supports $\Lambda$-cosmology); however, this result holds as long as the underlying cosmological model corresponds (or, is close enough) to the model adopted for generating the $\mathrm{GW}$ mock data.

Last but not least, considering the upcoming cosmological surveys, such as Simons Observatory Collaboration, CMB Stage-4, DESI, LSST, weak lensing, and galaxy clusters, it is quite reasonable to examine the constraining power of the Einstein Telescope compared to others. A systematic and dedicated analysis for dynamical dark energy is the subject of a forthcoming work.

\section{ACKNOWLEDGMENTS}

The authors gratefully acknowledge the referee for several essential comments that improved the manuscript significantly. W. Y. was supported by the National Natural Science Foundation of China under Grants No. 11705079 and No. 11647153 . The work of L. X. is supported in part by National Natural Science Foundation of China under Grant No. 11675032. S. P. acknowledges the financial support coming from the Faculty Research and Professional Development Fund (FRPDF) Scheme of Presidency University, Kolkata, India. D. F. M. thanks the Research Council of Norway and the NOTUR computing facilities. This paper is based upon work from COST action Grant No. CA15117 (CANTATA), supported by COST (European Cooperation in Science and Technology).

\section{APPENDIX: BEST-FIT VALUES OF FREE AND DERIVED PARAMETERS OF THE DARK ENERGY PARAMETRIZATIONS}

In this section we show the best-fit values of the free and derived parameters of all the dark energy
TABLE VII. The table summarizes the best-fit values of the free and derived parameters of the CPL parametrization (10). Here, $\mathrm{CB}=\mathrm{CMB}+\mathrm{BAO}, \mathrm{CBJ}=\mathrm{CMB}+\mathrm{BAO}+\mathrm{JLA}$, and $\mathrm{CBJC}=\mathrm{CMB}+\mathrm{BAO}+\mathrm{JLA}+\mathrm{CC}$.

\begin{tabular}{lllll}
\hline \hline Parameters & CMB & CB & CBJ & CBJC \\
\hline$\Omega_{c} h^{2}$ & 0.1203 & 0.1194 & 0.1193 & 0.1189 \\
$\Omega_{b} h^{2}$ & 0.02232 & 0.02218 & 0.02227 & 0.02214 \\
$100 \theta_{M C}$ & 1.041 & 1.041 & 1.04055 & 1.04100 \\
$\tau$ & 0.060 & 0.051 & 0.0775 & 0.089 \\
$n_{s}$ & 0.9628 & 0.964 & 0.9657 & 0.967 \\
$\ln \left(10{ }^{10} A_{s}\right)$ & 3.060 & 3.038 & 3.082 & 3.108 \\
$w_{0}$ & -1.905 & -0.284 & -0.885 & -0.873 \\
$w_{a}$ & -0.083 & -1.927 & -0.518 & -0.510 \\
$\Omega_{m 0}$ & 0.149 & 0.376 & 0.308 & 0.308 \\
$\sigma_{8}$ & 1.072 & 0.760 & 0.834 & 0.841 \\
$H_{0}$ & 97.89 & 61.50 & 67.99 & 67.80 \\
\hline \hline
\end{tabular}

TABLE VIII. The table summarizes the best-fit values of the free and derived parameters of the logarithmic parametrization (11). Here, $\mathrm{CB}=\mathrm{CMB}+\mathrm{BAO}, \mathrm{CBJ}=\mathrm{CMB}+\mathrm{BAO}+\mathrm{JLA}$, and $\mathrm{CBJC}=\mathrm{CMB}+\mathrm{BAO}+\mathrm{JLA}+\mathrm{CC}$.

\begin{tabular}{lllll}
\hline \hline Parameters & CMB & \multicolumn{1}{c}{ CB } & \multicolumn{1}{c}{ CBJ } & CBJC \\
\hline$\Omega_{c} h^{2}$ & 0.1190 & 0.1198 & 0.1193 & 0.1194 \\
$\Omega_{b} h^{2}$ & 0.02215 & 0.02211 & 0.02237 & 0.02234 \\
$100 \theta_{M C}$ & 1.04082 & 1.04056 & 1.04058 & 1.04047 \\
$\tau$ & 0.083 & 0.080 & 0.089 & 0.084 \\
$n_{s}$ & 0.9650 & 0.9654 & 0.9688 & 0.9659 \\
$\ln \left(10{ }^{10} A_{s}\right)$ & 3.098 & 3.094 & 3.118 & 3.099 \\
$w_{0}$ & -1.413 & -0.651 & -0.872 & -0.908 \\
$w_{a}$ & -2.363 & -0.790 & -0.392 & -0.299 \\
$\Omega_{m 0}$ & 0.145 & 0.341 & 0.311 & 0.311 \\
$\sigma_{8}$ & 1.096 & 0.815 & 0.845 & 0.838 \\
$H_{0}$ & 98.89 & 64.70 & 67.65 & 67.67 \\
\hline \hline
\end{tabular}

TABLE IX. The table summarizes the best-fit values of the free and derived parameters of the JBP parametrization (12). Here, $\mathrm{CB}=\mathrm{CMB}+\mathrm{BAO}, \mathrm{CBJ}=\mathrm{CMB}+\mathrm{BAO}+\mathrm{JLA}$, and $\mathrm{CBJC}=\mathrm{CMB}+\mathrm{BAO}+\mathrm{JLA}+\mathrm{CC}$.

\begin{tabular}{lllll}
\hline \hline Parameters & $\mathrm{CMB}$ & $\mathrm{CB}$ & $\mathrm{CBJ}$ & $\mathrm{CBJC}$ \\
\hline$\Omega_{c} h^{2}$ & 0.1197 & 0.1203 & 0.1175 & 0.1188 \\
$\Omega_{b} h^{2}$ & 0.02234 & 0.02218 & 0.02242 & 0.02235 \\
$100 \theta_{M C}$ & 1.04103 & 1.04084 & 1.04095 & 1.04082 \\
$\tau$ & 0.078 & 0.091 & 0.082 & 0.085 \\
$n_{s}$ & 0.965 & 0.964 & 0.973 & 0.966 \\
$\ln \left(10^{10} A_{s}\right)$ & 3.086 & 3.120 & 3.090 & 3.107 \\
$w_{0}$ & -1.687 & -0.538 & -0.824 & -0.915 \\
$w_{a}$ & -0.752 & -2.335 & -0.870 & -0.499 \\
$\Omega_{m 0}$ & 0.162 & 0.344 & 0.311 & 0.310 \\
$\sigma_{8}$ & 1.052 & 0.823 & 0.815 & 0.832 \\
$H_{0}$ & 93.96 & 64.55 & 67.21 & 67.58 \\
\hline \hline
\end{tabular}


TABLE $X$. The table summarizes the best-fit values of the free and derived parameters of the BA parametrization (13). Here, $\mathrm{CB}=\mathrm{CMB}+\mathrm{BAO}, \mathrm{CBJ}=\mathrm{CMB}+\mathrm{BAO}+\mathrm{JLA}$, and $\mathrm{CBJC}=\mathrm{CMB}+\mathrm{BAO}+\mathrm{JLA}+\mathrm{CC}$.

\begin{tabular}{lcccc}
\hline \hline Parameters & $\mathrm{CMB}$ & $\mathrm{CB}$ & $\mathrm{CBJ}$ & $\mathrm{CBJC}$ \\
\hline$\Omega_{c} h^{2}$ & 0.1191 & 0.1183 & 0.1198 & 0.1191 \\
$\Omega_{b} h^{2}$ & 0.02239 & 0.02236 & 0.02222 & 0.02237 \\
$100 \theta_{M C}$ & 1.04095 & 1.04087 & 1.04100 & 1.04094 \\
$\tau$ & 0.096 & 0.076 & 0.076 & 0.089 \\
$n_{s}$ & 0.970 & 0.969 & 0.967 & 0.972 \\
$\ln \left(10^{10} A_{s}\right)$ & 3.118 & 3.082 & 3.084 & 3.118 \\
$w_{0}$ & -1.660 & -0.743 & -0.744 & -0.910 \\
$w_{a}$ & -0.486 & -0.395 & -0.511 & -0.133 \\
$\Omega_{m}$ & 0.156 & 0.327 & 0.322 & 0.318 \\
$\sigma_{8}$ & 1.078 & 0.806 & 0.826 & 0.834 \\
$H_{0}$ & 95.44 & 65.76 & 66.56 & 66.86 \\
\hline \hline
\end{tabular}

parametrizations that have been investigated in this work. Tables VII, VIII, IX, and X correspond to the CPL, logarithmic, JBP, and BA parametrization, respectively. We again note that the best-fit values of those parameters summarized in the aforementioned tables were used to generate the GW catalogue.
[1] B. P. Abbott et al. (LIGO Scientific and Virgo Collaborations), Observation of Gravitational Waves from a Binary Black Hole Merger, Phys. Rev. Lett. 116, 061102 (2016).

[2] B. P. Abbott et al. (LIGO Scientific and Virgo Collaborations), GW151226: Observation of Gravitational Waves from a 22-Solar-Mass Binary Black Hole Coalescence, Phys. Rev. Lett. 116, 241103 (2016).

[3] B. P. Abbott et al. (LIGO Scientific and VIRGO Collaborations), GW170104: Observation of a 50-Solar-Mass Binary Black Hole Coalescence at Redshift 0.2, Phys. Rev. Lett. 118, 221101 (2017).

[4] B. P. Abbott et al. (LIGO Scientific and Virgo Collaborations), GW170608: Observation of a 19-solar-mass binary black hole coalescence, Astrophys. J. 851, L35 (2017).

[5] B. P. Abbott et al. (LIGO Scientific and Virgo Collaborations), GW170814: A Three-Detector Observation of Gravitational Waves from a Binary Black Hole Coalescence, Phys. Rev. Lett. 119, 141101 (2017).

[6] B. P. Abbott et al. (LIGO Scientific and Virgo Collaborations), Upper limits on the rates of binary neutron star and neutron StarCblack hole mergers from advanced ligos first observing run, Astrophys. J. 832, L21 (2016).

[7] B. P. Abbott et al. (LIGO Scientific and Virgo Collaborations), GW170817: Observation of Gravitational Waves from a Binary Neutron Star Inspiral, Phys. Rev. Lett. 119, 161101 (2017).

[8] B. P. Abbott et al. (LIGO Scientific and Virgo Collaborations), Gravitational waves and gamma-rays from a binary neutron star merger: GW170817 and GRB 170817A, Astrophys. J. 848, L13 (2017).

[9] T. Baker, E. Bellini, P. G. Ferreira, M. Lagos, J. Noller, and I. Sawicki, Strong Constraints on Cosmological Gravity from GW170817 and GRB 170817A, Phys. Rev. Lett. 119, 251301 (2017).

[10] P. Creminelli and F. Vernizzi, Dark Energy after GW170817 and GRB170817A, Phys. Rev. Lett. 119, 251302 (2017).

[11] J. M. Ezquiaga and M. Zumalacárregui, Dark Energy After GW170817: Dead Ends and the Road Ahead, Phys. Rev. Lett. 119, 251304 (2017).

[12] J. Sakstein and B. Jain, Implications of the Neutron Star Merger GW170817 for Cosmological Scalar-Tensor Theories, Phys. Rev. Lett. 119, 251303 (2017).

[13] E. Di Valentino and A. Melchiorri, First cosmological constraints combining Planck with the recent gravitational-wave standard siren measurement of the Hubble constant, Phys. Rev. D 97, 041301 (2018).

[14] S. Chakraborty, K. Chakravarti, S. Bose, and S. SenGupta, Signatures of extra dimensions in gravitational waves from black hole quasinormal modes, Phys. Rev. D 97, 104053 (2018).

[15] L. Visinelli, N. Bolis, and S. Vagnozzi, Brane-world extra dimensions in light of GW170817, Phys. Rev. D 97, 064039 (2018).

[16] J. Oost, S. Mukohyama, and A. Wang, Constraints on Einstein-aether theory after GW170817, Phys. Rev. D 97, 124023 (2018).

[17] A. Casalino, M. Rinaldi, L. Sebastiani, and S. Vagnozzi, Mimicking dark matter and dark energy in a mimetic model compatible with GW170817, Phys. Dark Universe 22, 108 (2018).

[18] W. Zhao, B. S. Wright, and B. Li, Constraining the time variation of Newton's constant $G$ with gravitational-wave standard sirens and supernovae, J. Cosmol. Astropart. Phys. 10 (2018) 052. 
[19] T. Liu, X. Zhang, W. Zhao, K. Lin, C. Zhang, S. Zhang, X. Zhao, T. Zhu, and A. Wang, Waveforms of compact binary inspiral gravitational radiation in screened modified gravity, Phys. Rev. D 98, 083023 (2018).

[20] E. Di Valentino, D. E. Holz, A. Melchiorri, and F. Renzi, The cosmological impact of future constraints on $H_{0}$ from gravitational-wave standard sirens, Phys. Rev. D 98, 083523 (2018).

[21] J. J. Wei, Model-independent curvature determination from gravitational-wave standard sirens and cosmic chronometers, Astrophys. J. 868, 29 (2018).

[22] X. Zhang et al., Evidence of deviations from general relativity in binary pulsars, arXiv:1806.02581.

[23] J. M. Ezquiaga and M. Zumalacárregui, Dark energy in light of multi-messenger gravitational-wave astronomy, Front. Astron. Space Sci. 5, 44 (2018).

[24] R. Kase and S. Tsujikawa, Dark energy in Horndeski theories after GW170817: A review, Int. J. Mod. Phys. D 28, 1942005 (2019).

[25] P. Creminelli, M. Lewandowski, G. Tambalo, and F. Vernizzi, Gravitational wave decay into dark energy, J. Cosmol. Astropart. Phys. 12 (2018) 025.

[26] K. Lin, X. Zhao, C. Zhang, T. Liu, B. Wang, S. Zhang, X. Zhang, W. Zhao, T. Zhu, and A. Wang, Gravitational wave forms, polarizations, response functions and energy losses of triple systems in Einstein-Aether theory, Phys. Rev. D 99, 023010 (2019).

[27] R. C. Nunes, S. Pan, and E. N. Saridakis, New observational constraints on $f(T)$ gravity through gravitational-wave astronomy, Phys. Rev. D 98, 104055 (2018).

[28] E. J. Copeland, M. Kopp, A. Padilla, P. M. Saffin, and C. Skordis, Dark Energy after GW170817, Revisited, Phys. Rev. Lett. 122, 061301 (2019).

[29] A. Casalino, M. Rinaldi, L. Sebastiani, and S. Vagnozzi, Alive and well: Mimetic gravity and a higher-order extension in light of GW170817, Classical Quantum Gravity 36, 017001 (2019).

[30] M. Chevallier and D. Polarski, Accelerating universes with scaling dark matter, Int. J. Mod. Phys. D 10, 213 (2001).

[31] E. V. Linder, Exploring the Expansion History of the Universe, Phys. Rev. Lett. 90, 091301 (2003).

[32] A. R. Cooray and D. Huterer, Gravitational lensing as a probe of quintessence, Astrophys. J. 513, L95 (1999).

[33] P. Astier, Can luminosity distance measurements probe the equation of state of dark energy, Phys. Lett. B 500, 8 (2001).

[34] J. Weller and A. Albrecht, Future supernovae observations as a probe of dark energy, Phys. Rev. D 65, 103512 (2002).

[35] G. Efstathiou, Constraining the equation of state of the Universe from distant type Ia supernovae and cosmic microwave background anisotropies, Mon. Not. R. Astron. Soc. 310, 842 (1999).

[36] H. K. Jassal, J. S. Bagla, and T. Padmanabhan, Observational constraints on low redshift evolution of dark energy: How consistent are different observations, Phys. Rev. D 72 , 103503 (2005).

[37] E. V. Linder and D. Huterer, How many dark energy parameters, Phys. Rev. D 72, 043509 (2005).

[38] E. V. Linder, Biased Cosmology: Pivots, Parameters, and Figures of Merit, Astropart. Phys. 26, 102 (2006).
[39] E. M. Barboza, Jr. and J. S. Alcaniz, A parametric model for dark energy, Phys. Lett. B 666, 415 (2008).

[40] E. Di Valentino, A. Melchiorri, and J. Silk, Reconciling Planck with the local value of $H_{0}$ in extended parameter space, Phys. Lett. B 761, 242 (2016).

[41] W. Yang, R. C. Nunes, S. Pan, and D. F. Mota, Effects of neutrino mass hierarchies on dynamical dark energy models, Phys. Rev. D 95, 103522 (2017).

[42] E. Di Valentino, A. Melchiorri, E. V. Linder, and J. Silk, Constraining dark energy dynamics in extended parameter space, Phys. Rev. D 96, 023523 (2017).

[43] E. Di Valentino, Crack in the cosmological paradigm, Nat. Astron. 1, 569 (2017).

[44] W. Yang, S. Pan, and A. Paliathanasis, Latest astronomical constraints on some nonlinear parametric dark energy models, Mon. Not. R. Astron. Soc. 475, 2605 (2018).

[45] S. Pan, E. N. Saridakis, and W. Yang, Observational constraints on oscillating dark-energy parametrizations, Phys. Rev. D 98, 063510 (2018).

[46] S. Vagnozzi, S. Dhawan, M. Gerbino, K. Freese, A. Goobar, and O. Mena, Constraints on the sum of the neutrino masses in dynamical dark energy models with $w(z) \geq-1$ are tighter than those obtained in $\Lambda$ CDM, Phys. Rev. D 98, 083501 (2018).

[47] W. Yang, S. Pan, E. Di Valentino, E. N. Saridakis, and S. Chakraborty, Observational constraints on one-parameter dynamical dark-energy parametrizations and the $H_{0}$ tension, Phys. Rev. D 99, 043543 (2019).

[48] W. Yang, S. Pan, E. Di Valentino, and E. N. Saridakis, Observational constraints on dynamical dark energy with pivoting redshift, arXiv:1811.06932.

[49] R. Adam et al. (Planck Collaboration), Planck 2015 results. I. Overview of products and scientific results, Astron. Astrophys. 594, A1 (2016).

[50] N. Aghanim et al. (Planck Collaboration), Planck 2015 results. XI. CMB power spectra, likelihoods, and robustness of parameters, Astron. Astrophys. 594, A11 (2016).

[51] F. Beutler, C. Blake, M. Colless, D. H. Jones, L. StaveleySmith, L. Campbell, Q. Parker, W. Saunders, and F. Watson, The $6 \mathrm{dF}$ galaxy survey: Baryon acoustic oscillations and the local Hubble constant, Mon. Not. R. Astron. Soc. 416, 3017 (2011).

[52] A. J. Ross, L. Samushia, C. Howlett, W. J. Percival, A. Burden, and M. Manera, The clustering of the SDSS DR7 main Galaxy sample C I. A 4 per cent distance measure at $z=0.15$, Mon. Not. R. Astron. Soc. 449, 835 (2015).

[53] H. Gil-Marín et al., The clustering of galaxies in the SDSSIII baryon oscillation spectroscopic survey: BAO measurement from the LOS-dependent power spectrum of DR12 BOSS galaxies, Mon. Not. R. Astron. Soc. 460, 4210 (2016).

[54] M. Betoule et al. (SDSS Collaboration), Improved cosmological constraints from a joint analysis of the SDSS-II and SNLS supernova samples, Astron. Astrophys. 568, A22 (2014).

[55] M. Moresco, L. Pozzetti, A. Cimatti, R. Jimenez, C. Maraston, L. Verde, D. Thomas, A. Citro, R. Tojeiro, and D. Wilkinson, A $6 \%$ measurement of the Hubble parameter at $z \sim 0.45$ : Direct evidence of the epoch of cosmic reacceleration, J. Cosmol. Astropart. Phys. 05 (2016) 014. 
[56] W. Zhao, C. Van Den Broeck, D. Baskaran, and T. G. F. Li, Determination of dark energy by the Einstein telescope: Comparing with CMB, BAO and SNIa observations, Phys. Rev. D 83, 023005 (2011).

[57] R. G. Cai and T. Yang, Estimating cosmological parameters by the simulated data of gravitational waves from the Einstein Telescope, Phys. Rev. D 95, 044024 (2017).

[58] X. Zhang, J. Yu, T. Liu, W. Zhao, and A. Wang, Testing Brans-Dicke gravity using the Einstein telescope, Phys. Rev. D 95, 124008 (2017).

[59] R. G. Cai, T. B. Liu, X. W. Liu, S. J. Wang, and T. Yang, Probing cosmic anisotropy with gravitational waves as standard sirens, Phys. Rev. D 97, 103005 (2018).

[60] L.F. Wang, X. N. Zhang, J.F. Zhang, and X. Zhang, Impacts of gravitational-wave standard siren observation of the Einstein Telescope on weighing neutrinos in cosmology, Phys. Lett. B 782, 87 (2018).

[61] X. N. Zhang, L.F. Wang, J.F. Zhang, and X. Zhang, Improving cosmological parameter estimation with the future gravitational-wave standard siren observation from the Einstein Telescope, Phys. Rev. D 99, 063510 (2019).

[62] H. Audley et al. (LISA Collaboration), Laser interferometer space antenna, arXiv:1702.00786.

[63] S. Kawamura et al., The Japanese space gravitational wave antenna: DECIGO, Classical Quantum Gravity 28, 094011 (2011).

[64] S. Sato et al., The status of DECIGO, J. Phys. Conf. Ser. 840, 012010 (2017).

[65] J. Luo et al. (TianQin Collaboration), TianQin: A spaceborne gravitational wave detector, Classical Quantum Gravity 33, 035010 (2016).

[66] J. Aguirre et al. (Simons Observatory Collaboration), The Simons observatory: Science goals and forecasts, J. Cosmol. Astropart. Phys. 02 (2019) 056.

[67] M. H. Abitbol et al. (CMB-S4 Collaboration), CMB-S4 Technology Book, arXiv:1706.02464.

[68] R. Laureijs et al. (EUCLID Collaboration), Euclid definition study report, arXiv:1110.3193.

[69] R. Scaramella et al. (Euclid Collaboration), Euclid space mission: A cosmological challenge for the next 15 years, IAU Symp. 10, 375 (2014).

[70] A. Aghamousa et al. (DESI Collaboration), The DESI experiment part I: Science, targeting, and survey design, arXiv:1611.00036.

[71] J. A. Newman et al. (LSST Dark Energy Science Collaboration), Deep multi-object spectroscopy to enhance dark energy science from LSST, arXiv:1903.09325.

[72] R. A. Hložek et al. (LSST Dark Energy Science Collaboration), Single-object imaging and spectroscopy to enhance dark energy science from LSST, arXiv:1903.09324.

[73] R. Mandelbaum et al. (LSST Dark Energy Science Collaboration), Wide-field multi-object spectroscopy to enhance dark energy science from LSST, arXiv:1903.09323.

[74] P. A. R. Ade et al. (Planck Collaboration), Planck 2015 results. XIII. Cosmological parameters, Astron. Astrophys. 594, A13 (2016).

[75] V. F. Mukhanov, H. A. Feldman, and R. H. Brandenberger, Theory of cosmological perturbations, Phys. Rep. 215, 203 (1992).
[76] C. P. Ma and E. Bertschinger, Cosmological perturbation theory in the synchronous and conformal Newtonian gauges, Astrophys. J. 455, 7 (1995).

[77] K. A. Malik and D. Wands, Cosmological perturbations, Phys. Rep. 475, 1 (2009).

[78] W. Yang, S. Pan, L. Xu, and D. F. Mota, Effects of anisotropic stress in interacting dark matter-Dark energy scenarios, Mon. Not. R. Astron. Soc. 482, 1858 (2019).

[79] R. Schneider, V. Ferrari, S. Matarrese, and S. F. P. Zwart, Gravitational waves from cosmological compact binaries, Mon. Not. R. Astron. Soc. 324, 797 (2001).

[80] C. Cutler and D. E. Holz, Ultra-high precision cosmology from gravitational waves, Phys. Rev. D 80, 104009 (2009).

[81] T. G. F. Li, Extracting physics from gravitational waves, Springer theses, 2015.

[82] M. Abernathy et al. (ET Science Team), Einstein gravitational wave Telescope conceptual design study, Report No. ET-0106C-10 (issue: 4); see the website: www.et-gw .eu/.

[83] B. Sathyaprakash et al., Scientific objectives of Einstein Telescope, Classical Quantum Gravity 29, 124013 (2012); Erratum, Classical Quantum Gravity 30, 079501(E) (2013)

[84] T. Yang, R. F. L. Holanda, and B. Hu, Constraints on the cosmic distance duality relation with simulated data of gravitational waves from the Einstein Telescope, Astropart. Phys. 108, 57 (2019).

[85] Z. C. Chen, F. Huang, and Q. G. Huang, Stochastic gravitational-wave background from binary black holes and binary neutron stars and implications for LISA, Astrophys. J. 871, 97 (2019).

[86] G. Cusin, I. Dvorkin, C. Pitrou, and J. P. Uzan, First Predictions of the Angular Power Spectrum of the Astrophysical Gravitational Wave Background, Phys. Rev. Lett. 120, 231101 (2018).

[87] I. Dvorkin, J. P. Uzan, E. Vangioni, and J. Silk, Synthetic model of the gravitational wave background from evolving binary compact objects, Phys. Rev. D 94, 103011 (2016).

[88] B. P. Abbott et al. (LIGO Scientific and VIRGO Collaborations), GW170104: Observation of a 50-Solar-Mass Binary Black Hole Coalescence at Redshift 0.2, Phys. Rev. Lett. 118, 221101 (2017); Erratum, Phys. Rev. Lett. 121, 129901(E) (2018).

[89] B. P. Abbott et al. (LIGO Scientific and Virgo Collaborations), GW170817: Observation of Gravitational Waves from a Binary Neutron Star Inspiral, Phys. Rev. Lett. 119, 161101 (2017).

[90] A. Lewis and S. Bridle, Cosmological parameters from CMB and other data: A Monte Carlo approach, Phys. Rev. D 66, 103511 (2002).

[91] A. Lewis, Efficient sampling of fast and slow cosmological parameters, Phys. Rev. D 87, 103529 (2013).

[92] A. Gelman and D. Rubin, Inference from iterative simulation using multiple sequences, Stat. Sci. 7, 457 (1992).

[93] http://cosmologist.info/cosmomc/.

[94] D. M. Scolnic et al., The complete light-curve sample of spectroscopically confirmed SNe Ia from Pan-STARRS1 and cosmological constraints from the combined Pantheon sample, Astrophys. J. 859, 101 (2018). 